\title{
Exponential multistability of memristive Cohen-Grossberg neural networks with stochastic parameter perturbations
}

\author{
Wei Yao ${ }^{\mathrm{a}}$, Chunhua Wang ${ }^{\mathrm{a}, *}$, Yichuang Sun ${ }^{\mathrm{b}}$, Chao Zhou ${ }^{\mathrm{a}}$, Hairong Lin ${ }^{\mathrm{a}}$ \\ ${ }^{a}$ College of Information Science and Engineering, Hunan University, Changsha 410082, China \\ ${ }^{b}$ School of Engineering and Technology, University of Hertfordshire, Hatfield AL10 9AB, U.K
}

\begin{abstract}
Due to instability being induced easily by parameter disturbances of network systems, this paper investigates the multistability of memristive Cohen-Grossberg neural networks (MCGNNs) under stochastic parameter perturbations. It is demonstrated that stable equilibrium points of MCGNNs can be flexibly located in the odd-sequence or evensequence regions. Some sufficient conditions are derived to ensure the exponential multistability of MCGNNs under parameter perturbations. It is found that there exist at least $(w+2)^{l}\left(\right.$ or $\left.(w+1)^{l}\right)$ exponentially stable equilibrium points in the odd-sequence (or the even-sequence) regions. In the paper, two numerical examples are given to verify the correctness and effectiveness of the obtained results.
\end{abstract}

Keywords: exponential multistability, memristive Cohen-Grossberg neural network, stochastic parameter perturbation, stable equilibrium point

\section{Introduction}

In the past few decades, complex system including neural network (NN) has been widely investigated due to its widespread application [1-14]. In [4], a set of new sufficient criteria were presented to ensure the stability and the appearance of Hopf bifurcation of fractional-order neural network with multiple delays. Xu and Zhang investigated exponential stability of antiperiodic solutions of a class of Cohen-Grossberg shunting inhibitory neural networks with time-varying delays and impulses [6]. In [7], H-infinity filtering for discrete-time uncertain systems with quantized measurements and packet dropouts was discussed. A state feedback controller was provided to render stochastic feedforward nonlinear systems globally asymptotically stable [9]. Switching laws were designed to maintain the stability of delayed switched nonlinear systems with both stable and unstable modes [11]. In [12], a novel secondorder sliding mode controller was designed subject to an output constraint. Finite-time stabilization in probability was addressed for a class of high-order stochastic nonlinear systems with output constraints [13]. Because of the nonvolatility of memristor, memristor-based circuits and applications have attracted increasing attention [15-19]. By using memristor to emulate synapse, memristive neural network (MNN) can be built [20-22]. Currently, MNN has been extensively applied in some fields such as logical operations and image processing [21, 22].

Multistability, an important dynamical characteristic of neural networks, has been extensively investigated in recent years [23-41]. For example, Kaslik and Sivasundaram researched multistability of discrete-time Hopfield NNs with distributed delays and impulses [25]. In [26], Chen et al. studied multistability of stochastic delayed Hopfield NNs by applying geometrical configuration of activation function. Wang et al. [27] investigated multistability of almost-periodic solutions of delayed NNs considering two classes of activation function: one class of function is piecewise linear with two corner points and the other class of function is continuously nondecreasing. In [28], Cheng et al. analysed multistability of delayed NNs considering a typical class of activation function and piecewise linear activation function. In [29], Yang et al. researched multistability of discrete-time delayed Cohen-Grossberg NNs

\footnotetext{
${ }^{*}$ Corresponding author

Email address: wch1227164@hnu.edu.cn (Chunhua Wang)
} 
with activation function possessing 2 and $2 r$ corner points. In [30], Zeng et al. addressed multistability of recurrent NNs with time-varying delays and piecewise linear activation function. The activation function in [30] is divided into $4 k-1$ intervals by $4 k$ corner points, and $(2 k)^{n}$ stable equilibrium points can be obtained. In [31], the activation function is divided into $2 m+1$ intervals by $2 m$ corner points, and $(m+1)^{n}$ stable equilibrium points can be acquired by investigating multistability of recurrent NNs. It is worth noting that the activation function is symmetrical about the origin [31]. In [32], local stability of multiple equilibria of NNs was addressed. The piecewise linear activation function with $2 r$ corner points is nondecreasing and $(r+1)^{n}$ stable equilibria can be acquired [32]. Global stability of MNNs with monotone nondecreasing activation function was discussed in [33]. Wu and Zhang analyzed multistability of delayed MNNs with piecewise linear activation function possessing two corner points in [34]. In [35], Nie et al. researched stable equilibria of MNNs with time-varying delay and piecewise linear activation function having 4 corner points. In [41], dynamical and static multisynchronization of coupled multistable neural networks was investigated, and every subnetwork of coupled neural networks can acquire $(r+1)^{n}$ locally exponentially stable periodic orbits or equilibrium points. In 1984, Hopfield proposed a new class of neural network and pointed out that convergent flow to stable states is the essential feature of this content-addressable memory operation [42]. Moreover, if location of a stable point in state space is viewed as the information of a particular memory, states near to the stable point contain partial information about that memory [42]. Therefore, as many stable equilibrium states as possible and flexible regions of stable equilibrium points are very necessary for neural network systems in some applications [23-30, 39, $40,42]$, such as associative memory storage and image processing. Generally, stable equilibrium points of MNNs locate in the odd-sequence regions in the existing results [27-32, 34]. Thus, it is very challenging and meaningful to generate a mass of stable equilibrium points and change flexibly their ranges.

Cohen-Grossberg neural network which takes some famous neural networks and systems such as Hopfield neural networks and Lotka-Volterra system as its special cases, was first proposed by Cohen and Grossberg [43, 44]. In the past few years, memristive Cohen-Grossberg neural network (MCGNNs) and its dynamical characteristics have been widely investigated [45-50]. In [45, 46], the exponential synchronization issue of MCGNNs with delays was discussed. Using the fixed point theorem, Nie et al. addressed the multistability topic of MCGNNs and obtained $3^{n}$ exponentially stable equilibrium points for $n$-dimensional MCGNNs with piecewise linear activation functions [47]. Multistability of MCGNNs with mixed delays was discussed and multiple almost periodic solutions were obtained in [49]. In [50], global exponential stability of MCGNNs with mixed delays and impulse perturbations was investigated. Due to dependence on state for parameters of MCGNNs and environment disturbances, parameter perturbations exist inevitably in the systems in reality. Parameter perturbations may lead to instability and some unpredictable influence for the systems. Therefore, the effect of parameter perturbations cannot be ignored. In addition, time delays especially time-varying delays will inevitably occur in the signal transmission of neurons because of the finite switching speed of amplifiers and neurons. In recent years, there are some researches on dynamical characteristics of MNNs under parameter perturbations [51-54]. However, there is no work on multistability of delayed MCGNNs with parameter perturbations. Achieving the multistability of memristive Cohen-Grossberg neural networks means that multistability of multiple classes of neural networks can be obtained. When multistability is exponential, systems can acquire fast convergence speed and good performance.

Inspired by the discussion above, this paper investigates the exponential multistability of MCGNNs with timevarying delays and parameter perturbations. We summarize the main contributions as follows.

1) Considering some unpredictable factors of environment, this paper investigates the multistability of MCGNNs with stochastic parameter perturbations for the first time.

2) Some sufficient conditions are presented to guarantee exponential multistability of MCGNNs with time-varying delays and parameter perturbations.

3) The exponentially stable equilibrium points of MCGNN system can be flexibly located in the odd-sequence or the even-sequence regions. And there exist at least $(w+2)^{l}\left(\right.$ or $\left.(w+1)^{l}\right)$ exponentially stable equilibrium points in the odd-sequence (or the even-sequence) regions. This means that the perturbed MCGNN system has potential application value in associative memory storage and secure communication. Therefore, the obtained result can enlarge and strengthen the existing results.

The rest of the paper is organized as follows. In Section 2, MCGNNs with time-varying delays and parameter perturbations are introduced. Some sufficient conditions are obtained to achieve exponential multistability of MCGNNs in Section 3. Section 4 presents numerical simulations to verify the effectiveness of the obtained results. Finally, conclusions are given in Section 5. 


\section{Preliminaries}

We consider memristive Cohen-Grossberg neural networks (MCGNNs) with time-varying delays as follows.

$$
\begin{aligned}
& \dot{p}_{m}(t)=a_{m}\left(p_{m}(t)\right)\left\{-b_{m} p_{m}(t)+\sum_{z=1}^{l} c_{m z}\left(p_{m}(t)\right) f_{z}\left(p_{z}(t)\right)\right. \\
& \left.+\sum_{z=1}^{l} d_{m z}\left(p_{m}(t)\right) f_{z}\left(p_{z}\left(t-\tau_{m z}(t)\right)\right)+I_{m}\right\}, m=1,2, \ldots, l,
\end{aligned}
$$

where $p_{m}(t)$ denotes the state of the $m$ th neuron; $a_{m}($.$) is amplification function; c_{m z}($.$) and d_{m z}($.$) represent memristive$ connection weights; $f_{z}($.$) is the activation function; \tau_{m z}(t)$ denotes time-varying delay and satisfies $0 \leq \tau_{m z}(t) \leq \tau$, where $\tau$ is a positive constant; $I_{m}$ is external input. We denote memristive connection weights as

$$
\begin{gathered}
c_{m z}\left(p_{m}(t)\right)= \begin{cases}c_{m z}^{(1)}, & \left|p_{m}(t)\right| \leq \chi_{m}, \\
c_{m z}^{(2)}, & \left|p_{m}(t)\right|>\chi_{m},\end{cases} \\
d_{m z}\left(p_{m}(t)\right)= \begin{cases}d_{m z}^{(1)}, & \left|p_{m}(t)\right| \leq \chi_{m}, \\
d_{m z}^{(2)}, & \left|p_{m}(t)\right|>\chi_{m},\end{cases}
\end{gathered}
$$

where $c_{m z}^{(1)}, c_{m z}^{(2)}, d_{m z}^{(1)}$ and $d_{m z}^{(2)}$ are constants, $\chi_{m}>0$ is the switching jump.

We denote $\hat{c}_{m z}=\max \left\{\left|c_{m z}^{(1)}\right|,\left|c_{m z}^{(2)}\right|\right\}, \bar{c}_{m z}=\max \left\{c_{m z}^{(1)}, c_{m z}^{(2)}\right\}, \tilde{c}_{m z}=\min \left\{c_{m z}^{(1)}, c_{m z}^{(2)}\right\}, \hat{d}_{m z}=\max \left\{\left|d_{m z}^{(1)}\right|,\left|d_{m z}^{(2)}\right|\right\}, \bar{d}_{m z}=\max \left\{d_{m z}^{(1)}, d_{m z}^{(2)}\right\}$ and $\tilde{d}_{m z}=\min \left\{d_{m z}^{(1)}, d_{m z}^{(2)}\right\}$.

Because of dependence on state for parameters of MCGNNs and environment disturbances, there unavoidably exist parameter perturbations in the systems in reality. Due to some unpredictable factors, parameter perturbations may randomly change along with time, which is called stochastic parameter perturbations. In this paper, we consider the multistability of MCGNNs with stochastic parameter perturbations. Accordingly, the MCGNN system with stochastic parameter perturbations can be written as

$$
\begin{aligned}
& \dot{p}_{m}(t)=a_{m}\left(p_{m}(t)\right)\left\{-b_{m} p_{m}(t)+\sum_{z=1}^{l}\left[c_{m z}\left(p_{m}(t)\right)+\Delta c_{m z}(t)\right] f_{z}\left(p_{z}(t)\right)\right. \\
& \left.+\sum_{z=1}^{l}\left[d_{m z}\left(p_{m}(t)\right)+\Delta d_{m z}(t)\right] f_{z}\left(p_{z}\left(t-\tau_{m z}(t)\right)\right)+I_{m}\right\}, m=1,2, \ldots, l,
\end{aligned}
$$

where $\Delta c_{m z}(t)$ and $\Delta d_{m z}(t)$ are stochastic parameters.

For a given set $\gamma \subset \mathfrak{R}, c o[\gamma]$ represents the closure of the convex hull for set $\gamma$. Thus, we can have

$$
\begin{aligned}
& c o\left[c_{m z}\left(p_{m}(t)\right)\right]= \begin{cases}c_{m z}^{(1)}, & \left|p_{m}(t)\right|<\chi_{m}, \\
{\left[\tilde{c}_{m z}, \bar{c}_{m z}\right],} & \left|p_{m}(t)\right|=\chi_{m} \\
c_{m z}^{(2)}, & \left|p_{m}(t)\right|>\chi_{m},\end{cases} \\
& c o\left[d_{m z}\left(p_{m}(t)\right)\right]= \begin{cases}d_{m z}^{(1)}, & \left|p_{m}(t)\right|<\chi_{m}, \\
{\left[\tilde{d}_{m z}, \bar{d}_{m z}\right],} & \left|p_{m}(t)\right|=\chi_{m} \\
d_{m z}^{(2)}, & \left|p_{m}(t)\right|>\chi_{m},\end{cases}
\end{aligned}
$$

According to the theory of differential inclusion [55], the MCGNN system (2) can be rewritten as

$$
\begin{aligned}
& \dot{p}_{m}(t) \in a_{m}\left(p_{m}(t)\right)\left\{-b_{m} p_{m}(t)+\sum_{z=1}^{l}\left[\operatorname{co}\left[c_{m z}\left(p_{m}(t)\right)\right]+\Delta c_{m z}(t)\right] f_{z}\left(p_{z}(t)\right)\right. \\
& \left.+\sum_{z=1}^{l}\left[c o\left[d_{m z}\left(p_{m}(t)\right)\right]+\Delta d_{m z}(t)\right] f_{z}\left(p_{z}\left(t-\tau_{m z}(t)\right)\right)+I_{m}\right\}, m=1,2, \ldots, l,
\end{aligned}
$$

or equivalently, by the measurable selection theorem in [55], there exist measurable functions $c_{m z}^{*}\left(p_{m}(t)\right) \in \operatorname{co}\left[c_{m z}\left(p_{m}(t)\right)\right]$, $d_{m z}^{*}\left(p_{m}(t)\right) \in \operatorname{co}\left[d_{m z}\left(p_{m}(t)\right)\right]$, such that

$$
\begin{aligned}
& \dot{p}_{m}(t)=a_{m}\left(p_{m}(t)\right)\left\{-b_{m} p_{m}(t)+\sum_{z=1}^{l}\left[c_{m z}^{*}\left(p_{m}(t)\right)+\Delta c_{m z}(t)\right] f_{z}\left(p_{z}(t)\right)\right. \\
& \left.+\sum_{z=1}^{l}\left[d_{m z}^{*}\left(p_{m}(t)\right)+\Delta d_{m z}(t)\right] f_{z}\left(p_{z}\left(t-\tau_{m z}(t)\right)\right)+I_{m}\right\}, m=1,2, \ldots, l,
\end{aligned}
$$


We consider the initial condition of system (2) as

$$
p_{m}(s)=\Upsilon_{m}^{(1)}(s), s \in[-\tau, 0]
$$

We set constants $Q_{m}^{j}, U_{m}^{j}, j=0,1, \ldots, w$, which satisfy the following condition

$$
-\infty<Q_{m}^{0}<U_{m}^{0}<Q_{m}^{1}<\cdots<Q_{m}^{w}<U_{m}^{w}<+\infty .
$$

Denote

$$
\begin{gathered}
\left(-\infty, Q_{m}^{0}\right]=\left(-\infty, Q_{m}^{0}\right]^{1} \times\left(Q_{m}^{0}, U_{m}^{0}\right)^{0} \times \cdots \times\left[U_{m}^{w},+\infty\right)^{0}, \\
\left(Q_{m}^{0}, U_{m}^{0}\right)=\left(-\infty, Q_{m}^{0}\right]^{0} \times\left(Q_{m}^{0}, U_{m}^{0}\right)^{1} \times \cdots \times\left[U_{m}^{w},+\infty\right)^{0}, \\
\cdots \\
{\left[U_{m}^{w},+\infty\right)=\left(-\infty, Q_{m}^{0}\right]^{0} \times\left(Q_{m}^{0}, U_{m}^{0}\right)^{0} \times \cdots \times\left[U_{m}^{w},+\infty\right)^{1},}
\end{gathered}
$$

Then $\mathfrak{R}^{l}$ is divided into $(2 w+3)^{l}$ parts, namely,

$$
\begin{aligned}
\Theta= & \left\{\prod_{m=1}^{l}\left(-\infty, Q_{m}^{0}\right]^{\varepsilon_{m}^{1}} \times\left(Q_{m}^{0}, U_{m}^{0}\right)^{\varepsilon_{m}^{2}} \times \cdots \times\left[U_{m}^{w},+\infty\right)^{\varepsilon_{m}^{2 w+3}} \mid\left(\varepsilon_{m}^{1}, \varepsilon_{m}^{2}, \ldots, \varepsilon_{m}^{2 w+3}\right)\right. \\
& =(1,0, \ldots, 0) \operatorname{or}(0,1, \ldots, 0) \operatorname{or} \ldots \operatorname{or}(0,0, \ldots, 1)\} .
\end{aligned}
$$

We choose two subsets $\Theta_{1}$ and $\Theta_{2}$ given by

$$
\begin{aligned}
\Theta_{1}= & \left\{\prod_{m=1}^{l}\left(-\infty, Q_{m}^{0}\right]^{\varepsilon_{m}^{1}} \times\left(Q_{m}^{0}, U_{m}^{0}\right)^{0} \times\left[U_{m}^{0}, Q_{m}^{1}\right]^{\varepsilon_{m}^{2}} \times \cdots \times\left(Q_{m}^{w}, U_{m}^{w}\right)^{0} \times\left[U_{m}^{w},+\infty\right)^{\varepsilon_{m}^{w+2}} \mid\right. \\
& \left.\left(\varepsilon_{m}^{1}, \varepsilon_{m}^{2}, \ldots, \varepsilon_{m}^{w+2}\right)=(1,0, \ldots, 0) \operatorname{or}(0,1, \ldots, 0) \operatorname{or} \ldots \operatorname{or}(0,0, \ldots, 1)\right\}
\end{aligned}
$$

and

$$
\begin{aligned}
\Theta_{2}= & \left\{\prod_{m=1}^{l}\left(-\infty, Q_{m}^{0}\right]^{0} \times\left(Q_{m}^{0}, U_{m}^{0}\right)^{\varepsilon_{m}^{1}} \times\left[U_{m}^{0}, Q_{m}^{1}\right]^{0} \times \cdots \times\left(Q_{m}^{w}, U_{m}^{w}\right)^{\varepsilon_{m}^{w+1}} \times\left[U_{m}^{w},+\infty\right)^{0} \mid\right. \\
& \left.\left(\varepsilon_{m}^{1}, \varepsilon_{m}^{2}, \ldots, \varepsilon_{m}^{w+1}\right)=(1,0, \ldots, 0) \operatorname{or}(0,1, \ldots, 0) \operatorname{or} \ldots \operatorname{or}(0,0, \ldots, 1)\right\} .
\end{aligned}
$$

The following assumptions will be used in this paper.

Assumption 1: Amplification function $a_{m}\left(p_{m}(t)\right)$ is continuous and bounded, namely, there exist two positive constants $a_{m}^{(1)}$ and $a_{m}^{(2)}$, such that $0<a_{m}^{(1)} \leq a_{m}(x) \leq a_{m}^{(2)}$ for $\forall x \in \mathfrak{R}$.

Assumption 2: Parameter $b_{m}$ satisfies $a_{m}(x) b_{m}>0$, for $\forall x \in \mathfrak{R}, m=1,2, \ldots, l$.

Assumption 3: Activation function $f_{z}($.$) is bounded and satisfies Lipschitz condition, which means there exist$ constants $M_{z}^{(1)}, M_{z}^{(2)}, L_{z}^{(1)}, L_{z}^{(2)}$ such that $\min \left\{f_{z}(s)\right\}=M_{z}^{(1)}$ and $\max \left\{f_{z}(s)\right\}=M_{z}^{(2)}$ for $\forall s \in \mathfrak{R}$, where $M_{z}^{(1)}<M_{z}^{(2)}$, and $\left|f_{z}\left(\pi_{1}\right)-f_{z}\left(\pi_{2}\right)\right| \leq L_{z}^{(1)}\left|\pi_{1}-\pi_{2}\right|$ for any $\pi_{1}, \pi_{2} \in \Theta_{1}$, and $\left|f_{z}\left(\pi_{3}\right)-f_{z}\left(\pi_{4}\right)\right| \leq L_{z}^{(2)}\left|\pi_{3}-\pi_{4}\right|$ for any $\pi_{3}, \pi_{4} \in \Theta_{2}$.

Assumption 4: Stochastic parameter perturbations $\Delta c_{m z}(t)$ and $\Delta d_{m z}(t)$ are continuous and bounded, that is $\alpha_{m z}^{(1)} \leq$ $\Delta c_{m z}(t) \leq \alpha_{m z}^{(2)}$ and $\beta_{m z}^{(1)} \leq \Delta d_{m z}(t) \leq \beta_{m z}^{(2)}$, where $\alpha_{m z}^{(1)}, \alpha_{m z}^{(2)}, \beta_{m z}^{(1)}$ and $\beta_{m z}^{(2)}$ are constants. Moreover, there exists a constant $t_{m z}^{*}>0$, such that $\Delta c_{m z}(t)=\alpha_{m z}, \Delta d_{m z}(t)=\beta_{m z}$, for $\forall t \in\left[t_{m z}^{*},+\infty\right)$, where constants $\alpha_{m z} \in\left[\alpha_{m z}^{(1)}, \alpha_{m z}^{(2)}\right]$ and $\beta_{m z}$ $\in\left[\beta_{m z}^{(1)}, \beta_{m z}^{(2)}\right]$.

We denote $\hat{\alpha}_{m z}=\max \left\{\left|\alpha_{m z}^{(1)}\right|,\left|\alpha_{m z}^{(2)}\right|\right\}$ and $\hat{\beta}_{m z}=\max \left\{\left|\beta_{m z}^{(1)}\right|,\left|\beta_{m z}^{(2)}\right|\right\}$

Definition 1: There exists a constant vector $p^{\#}=\left(p_{1}^{\#}, p_{2}^{\#}, \ldots, p_{l}^{\#}\right)^{T}$, such that

$$
a_{m}\left(p_{m}^{\#}\right)\left\{-b_{m} p_{m}^{\#}+\sum_{z=1}^{l} c_{m z}\left(p_{m}^{\#}\right) f_{z}\left(p_{z}^{\#}\right)+\sum_{z=1}^{l} d_{m z}\left(p_{m}^{\#}\right) f_{z}\left(p_{z}^{\#}\right)+I_{m}\right\}=0,
$$

then $p^{\#}=\left(p_{1}^{\#}, p_{2}^{\#}, \ldots, p_{l}^{\#}\right)^{T}$ is an equilibrium point of MCGNN system (1), where $I_{m}$ is constant. 
Remark 1: According to the assumption 1, we can obtain that $p^{\#}=\left(p_{1}^{\#}, p_{2}^{\#}, \ldots, p_{l}^{\#}\right)^{T}$ is an equilibrium point of the MCGNN system (1) if the following condition

$$
-b_{m} p_{m}^{\#}+\sum_{z=1}^{l} c_{m z}\left(p_{m}^{\#}\right) f_{z}\left(p_{z}^{\#}\right)+\sum_{z=1}^{l} d_{m z}\left(p_{m}^{\#}\right) f_{z}\left(p_{z}^{\#}\right)+I_{m}=0
$$

holds.

From assumption 1, there exists antiderivative of $\frac{1}{a_{m}\left(p_{m}\right)}$. Choose such an antiderivative $r_{m}\left(p_{m}\right)$ which satisfies $r_{m}(0)=0$. Then $\frac{d}{d p_{m}} r_{m}\left(p_{m}\right)=\frac{1}{a_{m}\left(p_{m}\right)}$. Using the derivative theorem for inverse function, the inverse function $r_{m}^{-1}\left(x_{m}\right)$ of $r_{m}\left(p_{m}\right)$ is differentiable and $\frac{d}{d x_{m}} r_{m}^{-1}\left(x_{m}\right)=a_{m}\left(p_{m}\right)$, where $x_{m}=r_{m}\left(p_{m}\right)$. Setting $q_{m}(t)=r_{m}\left(p_{m}(t)\right)$, then we can get $\dot{q}_{m}(t)=\frac{\dot{p}_{m}(t)}{a_{m}\left(p_{m}(t)\right)}$, where $p_{m}(t)=r_{m}^{-1}\left(q_{m}(t)\right)$. Substituting these equalities into system (3), we can obtain the MCGNN system with stochastic parameter perturbations as follows

$$
\begin{aligned}
& \dot{q}_{m}(t) \in-b_{m} r_{m}^{-1}\left(q_{m}(t)\right)+\sum_{z=1}^{l}\left[\operatorname{co}\left[c_{m z}\left(r_{m}^{-1}\left(q_{m}(t)\right)\right)\right]+\Delta c_{m z}(t)\right] f_{z}\left(r_{z}^{-1}\left(q_{z}(t)\right)\right) \\
& +\sum_{z=1}^{l}\left[c o\left[d_{m z}\left(r_{m}^{-1}\left(q_{m}(t)\right)\right)\right]+\Delta d_{m z}(t)\right] f_{z}\left(r_{z}^{-1}\left(q_{z}\left(t-\tau_{m z}(t)\right)\right)\right)+I_{m}, m=1,2, \ldots, l .
\end{aligned}
$$

Lemma 1 [56]: If $f_{z}\left( \pm \chi_{z}\right)=0, z=1,2, \ldots, l$, we have

$$
\begin{aligned}
& \left|c o\left[c_{m z}\left(p_{m}(t)\right)\right] f_{z}\left(p_{z}(t)\right)-c o\left[c_{m z}\left(p_{m}^{\#}\right)\right] f_{z}\left(p_{z}^{\#}\right)\right| \\
& \leq \hat{c}_{m z} L_{z}\left|p_{z}(t)-p_{z}^{\#}\right|, \quad m, z=1,2, \ldots, l,
\end{aligned}
$$

and

$$
\begin{aligned}
& \left|c o\left[d_{m z}\left(p_{m}(t)\right)\right] f_{z}\left(p_{z}\left(t-\tau_{m z}(t)\right)\right)-c o\left[c_{m z}\left(p_{m}^{\#}\right)\right] f_{z}\left(p_{z}^{\#}\right)\right| \\
& \leq \hat{d}_{m z} L_{z}\left|p_{z}\left(t-\tau_{m z}(t)\right)-p_{z}^{\#}\right|, \quad m, z=1,2, \ldots, l .
\end{aligned}
$$

where $L_{z}=\max \left\{L_{z}^{(1)}, L_{z}^{(2)}\right\}$.

Definition 2: The equilibrium point $p^{\#}=\left(p_{1}^{\#}, p_{2}^{\#}, \ldots, p_{l}^{\#}\right)^{T}$ of MCGNN (2) is exponentially stable, if there exist positive constants $\alpha, \beta$, such that

$$
\left\|p(t)-p^{\#}\right\| \leq \alpha\left\|\Upsilon^{(1)}-p^{\#}\right\| e^{-\beta t}
$$

holds for any $t \geq 0$, where $p(t)=\left(p_{1}(t), p_{2}(t), \ldots, p_{l}(t)\right)^{T}$ is the solution of the disturbed MCGNN system (2) with initial value $\Upsilon^{(1)}=\left(\Upsilon_{1}^{(1)}(s), \Upsilon_{2}^{(1)}(s), \ldots, \Upsilon_{l}^{(1)}(s)\right)^{T}, s \in[-\tau, 0]$

Definition 3: If there exist at least two different equilibrium points $p^{\#}=\left(p_{1}^{\#}, p_{2}^{\#}, \ldots, p_{l}^{\#}\right)^{T}$ and $p^{*}=\left(p_{1}^{*}, p_{2}^{*}, \ldots, p_{l}^{*}\right)^{T}$ for MCGNN (2) which satisfy definition 2 with the corresponding different initial conditions $\Upsilon^{(1)}=\left(\Upsilon_{1}^{(1)}(s), \Upsilon_{2}^{(1)}(s)\right.$, $\left.\ldots, \Upsilon_{l}^{(1)}(s)\right)^{T}$ and $\Upsilon^{(2)}=\left(\Upsilon_{1}^{(2)}(s), \Upsilon_{2}^{(2)}(s), \ldots, \Upsilon_{l}^{(2)}(s)\right)^{T}$, then exponential multistability of MCGNN (2) can be achieved, i.e., the multistability of MCGNN (2) is exponential.

\section{Multistability of perturbed memristive Cohen-Grossberg neural networks}

In this section, we will discuss the exponential multistability problem of the perturbed MCGNN systems (2).

Theorem 1: The perturbed MCGNN system $(2)$ has at least $(w+2)^{l}$ exponentially stable equilibrium points in $\mathfrak{R}^{l}$ and $\Theta_{1}$ is a positive invariant set, if $f_{m}\left( \pm \chi_{m}\right)=0$ and assumptions 1-4 and the following conditions

$$
\begin{aligned}
& \mathrm{K}_{1}=-b_{m} Q_{m}^{j}+\max \left\{\tilde{c}_{m m} f_{m}\left(Q_{m}^{j}\right), \bar{c}_{m m} f_{m}\left(Q_{m}^{j}\right)\right\} \\
& +\max \left\{\alpha_{m m}^{(1)} f_{m}\left(Q_{m}^{j}\right), \alpha_{m m}^{(2)} f_{m}\left(Q_{m}^{j}\right)\right\}+\Phi_{m}^{1}+\Phi_{m}^{2}+I_{m}<0, \\
& \mathrm{~K}_{2}=-b_{m} U_{m}^{j}+\min \left\{\tilde{c}_{m m} f_{m}\left(U_{m}^{j}\right), \bar{c}_{m m} f_{m}\left(U_{m}^{j}\right)\right\} \\
& +\min \left\{\alpha_{m m}^{(1)} f_{m}\left(U_{m}^{j}\right), \alpha_{m m}^{(2)} f_{m}\left(U_{m}^{j}\right)\right\}+\Gamma_{m}^{1}+\Gamma_{m}^{2}+I_{m}>0, \\
& b_{m} a_{m}^{(1)}-\sum_{z=1}^{l}\left(\hat{c}_{m z}+\hat{\alpha}_{m z}\right) L_{z}^{(1)} a_{z}^{(2)}-\sum_{z=1}^{l}\left(\hat{d}_{m z}+\hat{\beta}_{m z}\right) L_{z}^{(1)} a_{z}^{(2)}>0
\end{aligned}
$$


hold, where

$$
\begin{aligned}
\Phi_{m}^{1}= & \sum_{z=1, z \neq m}^{l} \max \left\{\left(\tilde{c}_{m z}+\alpha_{m z}^{(1)}\right) M_{z}^{(1)},\left(\bar{c}_{m z}+\alpha_{m z}^{(2)}\right) M_{z}^{(1)},\right. \\
& \left.\left(\tilde{c}_{m z}+\alpha_{m z}^{(1)}\right) M_{z}^{(2)},\left(\bar{c}_{m z}+\alpha_{m z}^{(2)}\right) M_{z}^{(2)}\right\}, \\
\Phi_{m}^{2}= & \sum_{z=1}^{l} \max \left\{\left(\tilde{d}_{m z}+\beta_{m z}^{(1)}\right) M_{z}^{(1)},\left(\bar{d}_{m z}+\beta_{m z}^{(2)}\right) M_{z}^{(1)},\right. \\
& \left.\left(\tilde{d}_{m z}+\beta_{m z}^{(1)}\right) M_{z}^{(2)},\left(\bar{d}_{m z}+\beta_{m z}^{(2)}\right) M_{z}^{(2)}\right\}, \\
\Gamma_{m}^{1}= & \sum_{z=1, z \neq m}^{l} \min \left\{\left(\tilde{c}_{m z}+\alpha_{m z}^{(1)}\right) M_{z}^{(1)},\left(\bar{c}_{m z}+\alpha_{m z}^{(2)}\right) M_{z}^{(1)},\right. \\
& \left.\left(\tilde{c}_{m z}+\alpha_{m z}^{(1)}\right) M_{z}^{(2)},\left(\bar{c}_{m z}+\alpha_{m z}^{(2)}\right) M_{z}^{(2)}\right\}, \\
\Gamma_{m}^{2}= & \sum_{z=1}^{l} \min \left\{\left(\tilde{d}_{m z}+\beta_{m z}^{(1)}\right) M_{z}^{(1)},\left(\bar{d}_{m z}+\beta_{m z}^{(2)}\right) M_{z}^{(1)},\right. \\
& \left.\left(\tilde{d}_{m z}+\beta_{m z}^{(1)}\right) M_{z}^{(2)},\left(\bar{d}_{m z}+\beta_{m z}^{(2)}\right) M_{z}^{(2)}\right\} .
\end{aligned}
$$

Proof:

1) First, we take an arbitrary region $\bar{\Theta}_{1}$ from $\Theta_{1}$ and define the following function.

$$
\begin{aligned}
& h_{m}\left(p_{m}(t)\right)=-b_{m} p_{m}(t)+\left[c_{m m}^{*}\left(p_{m}(t)\right)+\Delta c_{m m}(t)\right] f_{m}\left(p_{m}(t)\right)+\sum_{z=1, z \neq m}^{l}\left[c_{m z}^{*}\left(p_{m}(t)\right)\right. \\
& \left.+\Delta c_{m z}(t)\right] f_{z}\left(p_{z}(t)\right)+\sum_{z=1}^{l}\left[d_{m z}^{*}\left(p_{m}(t)\right)+\Delta d_{m z}(t)\right] f_{z}\left(p_{z}\left(t-\tau_{m z}(t)\right)\right)+I_{m}, m=1,2, \ldots, l,
\end{aligned}
$$

Denote $t^{(0)}=\max _{1 \leq m, z \leq l}\left\{t_{m z}^{*}\right\}$. Then for $t \in\left[t^{(0)},+\infty\right)$, we can rewrite the function $h_{m}\left(p_{m}(t)\right)$ as follows.

$$
\begin{aligned}
& h_{m}\left(p_{m}(t)\right)=-b_{m} p_{m}(t)+\left[c_{m m}^{*}\left(p_{m}(t)\right)+\alpha_{m m}\right] f_{m}\left(p_{m}(t)\right)+\sum_{z=1, z \neq m}^{l}\left[c_{m z}^{*}\left(p_{m}(t)\right)\right. \\
& \left.+\alpha_{m z}\right] f_{z}\left(p_{z}(t)\right)+\sum_{z=1}^{l}\left[d_{m z}^{*}\left(p_{m}(t)\right)+\beta_{m z}\right] f_{z}\left(p_{z}\left(t-\tau_{m z}(t)\right)\right)+I_{m}, m=1,2, \ldots, l,
\end{aligned}
$$

When $p_{m}(t)=Q_{m}^{j}$ or $p_{m}(t)=U_{m}^{j}$, we have

$$
\begin{aligned}
& h_{m}\left(Q_{m}^{j}\right)=-b_{m} Q_{m}^{j}+\left[c_{m m}^{*}\left(Q_{m}^{j}\right)+\alpha_{m m}\right] f_{m}\left(Q_{m}^{j}\right)+\sum_{z=1, z \neq m}^{l}\left[c_{m z}^{*}\left(Q_{m}^{j}\right)+\alpha_{m z}\right] f_{z}\left(p_{z}(t)\right) \\
& +\sum_{z=1}^{l}\left[d_{m z}^{*}\left(Q_{m}^{j}\right)+\beta_{m z}\right] f_{z}\left(p_{z}\left(t-\tau_{m z}(t)\right)\right)+I_{m} \\
& \leq-b_{m} Q_{m}^{j}+\max \left\{\tilde{c}_{m m} f_{m}\left(Q_{m}^{j}\right), \bar{c}_{m m} f_{m}\left(Q_{m}^{j}\right)\right\} \\
& +\max \left\{\alpha_{m m}^{(1)} f_{m}\left(Q_{m}^{j}\right), \alpha_{m m}^{(2)} f_{m}\left(Q_{m}^{j}\right)\right\}+\Phi_{m}^{1}+\Phi_{m}^{2}+I_{m}<0,
\end{aligned}
$$

and

$$
\begin{aligned}
& h_{m}\left(U_{m}^{j}\right)=-b_{m} U_{m}^{j}+\left[c_{m m}^{*}\left(U_{m}^{j}\right)+\alpha_{m m}\right] f_{m}\left(U_{m}^{j}\right)+\sum_{z=1, z \neq m}^{l}\left[c_{m z}^{*}\left(U_{m}^{j}\right)+\alpha_{m z}\right] f_{z}\left(p_{z}(t)\right) \\
& +\sum_{z=1}^{l}\left[d_{m z}^{*}\left(U_{m}^{j}\right)+\beta_{m z}\right] f_{z}\left(p_{z}\left(t-\tau_{m z}(t)\right)\right)+I_{m} \\
& \geq-b_{m} U_{m}^{j}+\min \left\{\tilde{c}_{m m} f_{m}\left(U_{m}^{j}\right), \bar{c}_{m m} f_{m}\left(U_{m}^{j}\right)\right\} \\
& +\min \left\{\alpha_{m m}^{(1)} f_{m}\left(U_{m}^{j}\right), \alpha_{m m}^{(2)} f_{m}\left(U_{m}^{j}\right)\right\}+\Gamma_{m}^{1}+\Gamma_{m}^{2}+I_{m}>0,
\end{aligned}
$$

$j=0,1, \ldots, w$. Because of the continuity of $h_{m}(z)$, there exists at least a point $\tilde{p}_{m} \in\left[U_{m}^{j}, Q_{m}^{j+1}\right]$, such that $h_{m}\left(\tilde{p}_{m}\right)=0$, where $j=0,1, \ldots, w-1$. Moreover, combining with assumptions 1 and 2, we can obtain $\lim _{z \rightarrow-\infty} h_{m}(z)=+\infty$ and $\lim _{z \rightarrow+\infty} h_{m}(z)=-\infty$. Thus, there exists at least a point $\tilde{p}_{m} \in\left(-\infty, Q_{m}^{0}\right]$, such that $h_{m}\left(\tilde{p}_{m}\right)=0$. And there exists at least a 
point $\tilde{p}_{m} \in\left[U_{m}^{w},+\infty\right)$, such that $h_{m}\left(\tilde{p}_{m}\right)=0$. As the subset $\Theta_{1}$ consists of $(w+2)^{l}$ parts, we can get that there exist at least $(w+2)^{l}$ equilibrium points for the perturbed MCGNN system (2) in $\mathfrak{R}^{l}$.

2) We set that $p_{m}(t)$ is the solution of the perturbed MCGNN system (2) with initial condition $\Upsilon_{m}^{(1)}(s) \in \bar{\Theta}_{1}$, $s \in[-\tau, 0]$, then we assert $p_{m}(t) \in \bar{\Theta}_{1}$ for any $t \geq 0$. If it is false, there exists $t_{0}>0$ so that $p_{m}\left(t_{0}\right)=Q_{m}^{j},\left.\dot{p}_{m}(t)\right|_{t=t_{0}}>0$ or $p_{m}\left(t_{0}\right)=U_{m}^{j},\left.\dot{p}_{m}(t)\right|_{t=t_{0}}<0$. In fact, when $p_{m}\left(t_{0}\right)=Q_{m}^{j}$, we have $\left.\dot{p}_{m}(t)\right|_{t=t_{0}} \leq a_{m}\left(Q_{m}^{j}\right) \mathrm{K}_{1}<0$. Moreover, we can obtain $\left.\dot{p}_{m}(t)\right|_{t=t_{0}} \geq a_{m}\left(U_{m}^{j}\right) \mathrm{K}_{2}>0$ when $p_{m}\left(t_{0}\right)=U_{m}^{j}$. So, it is contradictory. Therefore, $\Theta_{1}$ is a positive invariant set.

3) Denote a function as follows

$$
\kappa_{k}(v)=b_{k} a_{k}^{(1)}-v-\sum_{z=1}^{l}\left(\hat{c}_{k z}+\hat{\alpha}_{k z}\right) L_{z}^{(1)} a_{z}^{(2)}-e^{\nu \tau} \sum_{z=1}^{l}\left(\hat{d}_{k z}+\hat{\beta}_{k z}\right) L_{z}^{(1)} a_{z}^{(2)}
$$

it is clear that $\kappa_{k}(0)>0$, and there exists a sufficiently small positive constant $\beta$, such that $\kappa_{k}(\beta)>0, k=1,2, \ldots, l$.

Let $p(t)=\left(p_{1}(t), p_{2}(t), \ldots, p_{l}(t)\right)^{T}$ be the solution of the disturbed MCGNN system (2) with initial condition $\Upsilon_{m}^{(1)}(s) \in \bar{\Theta}_{1}$ and $g_{m}(t)=q_{m}(t)-q_{m}^{\#}$, where $q_{m}(t)=r_{m}\left(p_{m}(t)\right), q_{m}^{\#}=r_{m}\left(p_{m}^{\#}\right)$. By the set-valued maps theory, we can have

$$
\begin{aligned}
& \dot{g}_{m}(t) \in-b_{m}\left[r_{m}^{-1}\left(q_{m}(t)\right)-r_{m}^{-1}\left(q_{m}^{\#}\right)\right]+\sum_{z=1}^{l}\left[c o\left[c_{m z}\left(r_{m}^{-1}\left(q_{m}(t)\right)\right)\right]+\Delta c_{m z}(t)\right] f_{z}\left(r_{z}^{-1}\left(q_{z}(t)\right)\right) \\
& -\sum_{z=1}^{l}\left[c o\left[c_{m z}\left(r_{m}^{-1}\left(q_{m}^{\#}\right)\right)\right]+\Delta c_{m z}(t)\right] f_{z}\left(r_{z}^{-1}\left(q_{z}^{\#}\right)\right)+\sum_{z=1}^{l}\left[c o\left[d_{m z}\left(r_{m}^{-1}\left(q_{m}(t)\right)\right)\right]+\Delta d_{m z}(t)\right] \\
& \times f_{z}\left(r_{z}^{-1}\left(q_{z}\left(t-\tau_{m z}(t)\right)\right)\right)-\sum_{z=1}^{l}\left[c o\left[d_{m z}\left(r_{m}^{-1}\left(q_{m}^{\#}\right)\right)\right]+\Delta d_{m z}(t)\right] f_{z}\left(r_{z}^{-1}\left(q_{z}^{\#}\right)\right), m=1,2, \ldots, l .
\end{aligned}
$$

From Lemma 1, we can obtain

$$
\begin{aligned}
& \left|c o\left[c_{m z}\left(r_{m}^{-1}\left(q_{m}(t)\right)\right)\right] f_{z}\left(r_{z}^{-1}\left(q_{z}(t)\right)\right)-c o\left[c_{m z}\left(r_{m}^{-1}\left(q_{m}^{\#}\right)\right)\right] f_{z}\left(r_{z}^{-1}\left(q_{z}^{\#}\right)\right)\right| \\
& \leq \hat{c}_{m z} L_{z}^{(1)}\left|r_{z}^{-1}\left(q_{z}(t)\right)-r_{z}^{-1}\left(q_{z}^{\#}\right)\right| \leq \hat{c}_{m z} L_{z}^{(1)} a_{m}^{(2)}\left|g_{m}(t)\right|,
\end{aligned}
$$

and

$$
\begin{aligned}
& \left|c o\left[d_{m z}\left(r_{m}^{-1}\left(q_{m}(t)\right)\right)\right] f_{z}\left(r_{m}^{-1}\left(q_{m}\left(t-\tau_{m z}(t)\right)\right)\right)-\operatorname{co}\left[c_{m z}\left(r_{m}^{-1}\left(q_{m}^{\#}\right)\right)\right] f_{z}\left(r_{z}^{-1}\left(q_{z}^{\#}\right)\right)\right| \\
& \left.\leq \hat{d}_{m z} L L_{z}^{(1)}\left|r_{m}^{-1}\left(q_{m}\left(t-\tau_{m z}(t)\right)\right)-r_{z}^{-1}\left(q_{z}^{\#}\right)\right| \leq \hat{d}_{m z} L_{z}^{(1)} a_{m}^{(2)} \mid g_{m}\left(t-\tau_{m z}(t)\right)\right) \mid .
\end{aligned}
$$

Let $x_{m}(t)=e^{\beta t}\left|g_{m}(t)\right|$ and $\ell=\max _{1 \leq m \leq l}\left\{\left|q_{m}(0)-q_{m}^{\#}\right|\right\}$. We can obtain $x_{m}(0) \leq \ell$ for any $m=1,2, \ldots, l$. Then we will prove the following inequality

$$
x_{m}(t) \leq \ell, t>0, m=1,2, \ldots, l .
$$

Supposing (9) is invalid, then we can find a $k \in\{1,2, \ldots, l\}$ and $t_{1}$ for the first time $x_{k}\left(t_{1}\right)=\ell, \dot{x}_{k}\left(t_{1}\right)>0, x_{k}(t) \leq \ell$, $t \in\left[0, t_{1}\right) ; x_{j}(t) \leq \ell, t \in\left[0, t_{1}\right], j=1,2, \ldots, l, j \neq k$.

$$
\left.\frac{d x_{k}(t)}{d t}\right|_{t=t_{1}}=\beta x_{k}\left(t_{1}\right)+\left.e^{\beta t_{1}} \operatorname{sign}\left(g_{k}\left(t_{1}\right)\right) \frac{d g_{k}(t)}{d t}\right|_{t=t_{1}}
$$

Because $\frac{d}{d q_{m}(t)} r_{m}^{-1}\left(q_{m}(t)\right)=a_{m}\left(p_{m}(t)\right)>0$, we can obtain that

$$
\begin{aligned}
& \operatorname{sign}\left(g_{m}\left(t_{1}\right)\right)\left[b_{m} r_{m}^{-1}\left(q_{m}\left(t_{1}\right)\right)-b_{m} r_{m}^{-1}\left(q_{m}^{\#}\right)\right] \\
& =b_{m}\left|r_{m}^{-1}\left(q_{m}\left(t_{1}\right)\right)-r_{m}^{-1}\left(q_{m}^{\#}\right)\right| .
\end{aligned}
$$


Then,

$$
\begin{aligned}
& \left.\frac{d x_{k}(t)}{d t}\right|_{t=t_{1}} \leq \beta x_{k}\left(t_{1}\right)+e^{\beta t_{1}}\left\{-b_{k}\left|r_{k}^{-1}\left(q_{k}\left(t_{1}\right)\right)-r_{k}^{-1}\left(q_{k}^{\#}\right)\right|+\sum_{z=1}^{l} \hat{c}_{k z} L_{z}^{(1)} a_{z}^{(2)}\left|g_{z}\left(t_{1}\right)\right|\right. \\
& \left.+\sum_{z=1}^{l} \hat{\alpha}_{k z} L_{z}^{(1)} a_{z}^{(2)}\left|g_{z}\left(t_{1}\right)\right|+\sum_{z=1}^{l} \hat{d}_{k z} L_{z}^{(1)} a_{z}^{(2)}\left|g_{z}\left(t_{1}-\tau_{k z}\left(t_{1}\right)\right)\right|+\sum_{z=1}^{l} \hat{\beta}_{k z} L_{z}^{(1)} a_{z}^{(2)}\left|g_{z}\left(t_{1}-\tau_{k z}\left(t_{1}\right)\right)\right|\right\} \\
& \leq \beta x_{k}\left(t_{1}\right)+e^{\beta t_{1}}\left\{\left(-b_{k} a_{k}^{(1)}+\sum_{z=1}^{l}\left(\hat{c}_{k z}+\hat{\alpha}_{k z}\right) L_{z}^{(1)} a_{z}^{(2)}\right)\left|g_{z}\left(t_{1}\right)\right|+\sum_{z=1}^{l}\left(\hat{d}_{k z}+\hat{\beta}_{k z}\right) L_{z}^{(1)} a_{z}^{(2)}\left|g_{z}\left(t_{1}-\tau_{k z}\left(t_{1}\right)\right)\right|\right\} \\
& \leq\left(\beta-b_{k} a_{k}^{(1)}+\sum_{z=1}^{l}\left(\hat{c}_{k z}+\hat{\alpha}_{k z}\right) L_{z}^{(1)} a_{z}^{(2)}\right) x_{k}\left(t_{1}\right)+e^{\beta \tau} \sum_{z=1}^{l}\left(\hat{d}_{k z}+\hat{\beta}_{k z}\right) L_{z}^{(1)} a_{z}^{(2)} x_{k}\left(t_{1}-\tau_{k z}\left(t_{1}\right)\right) \\
& \leq\left(\beta-b_{k} a_{k}^{(1)}+\sum_{z=1}^{l}\left(\hat{c}_{k z}+\hat{\alpha}_{k z} L_{z}^{(1)} a_{z}^{(2)}+e^{\beta \tau} \sum_{z=1}^{l}\left(\hat{d}_{k z}+\hat{\beta}_{k z}\right) L_{z}^{(1)} a_{z}^{(2)}\right)\right. \\
& \times \max _{1 \leq m \leq l}\left\{\left|q_{m}(0)-q_{m}^{\#}\right|\right\}<0
\end{aligned}
$$

So, we can have

$$
x_{m}(t)=e^{\beta t}\left|q_{m}(t)-q_{m}^{\#}\right| \leq\left|q_{m}(0)-q_{m}^{\#}\right|,
$$

then

Accordingly, we obtain

$$
\frac{e^{\beta t}}{a_{m}^{(2)}}\left|p_{m}(t)-p_{m}^{\#}\right| \leq \frac{1}{a_{m}^{(1)}}\left|p_{m}(0)-p_{m}^{\#}\right| .
$$

$$
\left|p_{m}(t)-p_{m}^{\#}\right| \leq \frac{a_{m}^{(2)}}{a_{m}^{(1)}}\left|p_{m}(0)-p_{m}^{\#}\right| e^{-\beta t} .
$$

So,

$$
\left\|p(t)-p^{\#}\right\| \leq \alpha\left\|\Upsilon^{(1)}-p^{\#}\right\| e^{-\beta t}
$$

where $\alpha=\max _{1 \leq m \leq l}\left\{\frac{a_{m}^{(2)}}{a_{m}^{(1)}}\right\}$.

Therefore, the equilibrium point $p^{\#}=\left(p_{1}^{\#}, p_{2}^{\#}, \ldots, p_{l}^{\#}\right)^{T}$ of MCGNN (2) is exponentially stable. Further, the perturbed MCGNN system (2) can have at least $(w+2)^{l}$ exponentially stable equilibrium points in $\mathfrak{R}^{l}$. The proof is finished.

Remark 2: Multistability of system means that the system possesses multiple stable states, such as multiple stable equilibrium points [34-37] and multiple stable periodic orbits [38, 41]. This paper mainly investigates multiple stable equilibrium points of MCGNNs and further achieves that these equilibrium points are exponentially stable. The process of proof of Theorem 1 (and Theorem 2) can be simplified as follows. Firstly, this MCGNN system acquires multiple equilibrium points using fixed point theorem, which is represented in the step 1) of the proof for Theorem 1 (and Theorem 2). Then applying contradictory method, a positive invariant set can be obtained, namely, these equilibrium points will locate in the positive invariant set all the while. This is shown in the step 2) of the proof for Theorem 1 (and Theorem 2). Finally, we use set-valued maps theory and differential inequality method to prove that these equilibrium points are exponentially stable, which is provided in the step 3) of the proof for Theorem 1 (and Theorem 2).

Remark 3: From the conditions of Theorem 1, assumptions 1-4 are needed, which seems conservative. However, these assumptions are necessary and laconic. Firstly, amplification function $a_{m}\left(p_{m}(t)\right)$ of assumption 1 is used to decide which region stable equilibrium points locate in. When $a_{m}\left(p_{m}(t)\right)$ is positive (or negative), stable equilibrium points locate in odd-sequence (or even-sequence) regions. Moreover, to achieve that equilibrium points are exponential stable, namely, to achieve the step 3) of the proof for Theorem 1, assumption 2 is applied. In addition, assumption 3 on activation function is essential of Lemma 1. Finally, due to instability being induced easily by parameter perturbations of network systems, assumption 4 gives some constraint conditions of stochastic parameter perturbations to achieve the multistability of MCGNNs. The similar analysis for the assumptions of Theorem 2 and corollaries 1-2 can be obtained. Therefore, these assumptions in theorems and corollaries are very necessary. 
Remark 4: In [30-32, 34, 35, 47], the multistability of system was realized under the strict condition that the activation function is piecewise linear. In this paper, this strict condition is discarded in the Theorem 1. Our analytical derivations in the above and the first example to be presented in the following demonstrate the validity of our conditions. Therefore, compared with these existing results, our result is less conservative.

We assume that activation function is piecewise linear which is represented as

$$
f_{m}(s)=\left\{\begin{array}{cc}
M_{m}^{(1)}, & s \in\left(-\infty, Q_{m}^{0}\right] \\
\gamma_{m}^{0} s+u_{m}^{0}, & s \in\left(Q_{m}^{0}, U_{m}^{0}\right) \\
v_{m}^{0} s+n_{m}^{0}, & s \in\left[U_{m}^{0}, Q_{m}^{1}\right] \\
\vdots & \vdots \\
\gamma_{m}^{w} s+u_{m}^{w}, & s \in\left(Q_{m}^{w}, U_{m}^{w}\right) \\
M_{m}^{(2)}, & s \in\left[U_{m}^{w},+\infty\right)
\end{array}\right.
$$

where $\gamma_{m}^{j}, u_{m}^{j}$ for $j=0,1, \ldots, w, v_{m}^{j}, n_{m}^{j}$ for $j=0,1, \ldots, w-1$, are constants; $\gamma_{m}^{j}>0, v_{m}^{j}<0 ; \min \left\{f_{m}(s)\right\}=M_{m}^{(1)}$ and $\max \left\{f_{m}(s)\right\}=M_{m}^{(2)}$. Then, we can have the following corollary.

Corollary 1: The perturbed MCGNN system (2) has at least $(w+2)^{l}$ exponentially stable equilibrium points in $\mathfrak{R}^{l}$ and $\Theta_{1}$ is a positive invariant set, if $f_{m}\left( \pm \chi_{m}\right)=0$, assumptions $1,2,4$ and the conditions (6), (7), and

$$
b_{m} a_{m}^{(1)}-\sum_{z=1}^{l}\left(\hat{c}_{m z}+\hat{\alpha}_{m z}\right) L_{z}^{\max } a_{z}^{(2)}-\sum_{z=1}^{l}\left(\hat{d}_{m z}+\hat{\beta}_{m z}\right) L_{z}^{\max } a_{z}^{(2)}>0,
$$

hold, where $L_{z}^{\max }=\max \left\{\left|v_{z}^{0}\right|,\left|v_{z}^{1}\right|, \ldots,\left|v_{z}^{w-1}\right|\right\}$.

If amplification function $a_{m}\left(p_{m}(t)\right)$ is negative, namely, it satisfies the condition of assumption 5 , we can obtain Theorem 2.

Assumption 5: Amplification function $a_{m}\left(p_{m}(t)\right)$ is continuous, and there exist two negative constants $a_{m}^{(3)}$ and $a_{m}^{(4)}$, such that $a_{m}^{(3)} \leq a_{m}(x) \leq a_{m}^{(4)}<0$ for $\forall x \in \mathfrak{R}$.

Theorem 2: The perturbed MCGNN system (2) has at least $(w+1)^{l}$ exponentially stable equilibrium points in $\mathfrak{R}^{l}$ and $\Theta_{2}$ is a positive invariant set, if $f_{m}\left( \pm \chi_{m}\right)=0$, assumptions 2-5, the conditions (6), (7), and

$$
-\left|b_{m}\right| a_{m}^{(4)}+\sum_{z=1}^{l}\left(\hat{c}_{m z}+\hat{\alpha}_{m z}\right) L_{z}^{(2)} a_{z}^{(3)}+\sum_{z=1}^{l}\left(\hat{d}_{m z}+\hat{\beta}_{m z}\right) L_{z}^{(2)} a_{z}^{(3)}>0
$$

hold.

Proof:

1) First, we take an arbitrary region $\bar{\Theta}_{2}$ from $\Theta_{2}$

Amplification function $a_{m}\left(p_{m}(t)\right)$ is negative in the light of the assumption 5, so we can rewrite the perturbed MCGNN system (2) as follows.

$$
\begin{aligned}
& \dot{p}_{m}(t)=-a_{m}\left(p_{m}(t)\right)\left\{b_{m} p_{m}(t)-\sum_{z=1}^{l}\left[c_{m z}^{*}\left(p_{m}(t)\right)+\Delta c_{m z}(t)\right] f_{z}\left(p_{z}(t)\right)\right. \\
& \left.-\sum_{z=1}^{l}\left[d_{m z}^{*}\left(p_{m}(t)\right)+\Delta d_{m z}(t)\right] f_{z}\left(p_{z}\left(t-\tau_{m z}(t)\right)\right)-I_{m}\right\}, m=1,2, \ldots, l,
\end{aligned}
$$

We define the following function.

$$
\begin{aligned}
& G_{m}\left(p_{m}(t)\right)=-h_{m}\left(p_{m}(t)\right) \\
& =b_{m} p_{m}(t)-\left[c_{m m}^{*}\left(p_{m}(t)\right)+\Delta c_{m m}(t)\right] f_{m}\left(p_{m}(t)\right) \\
& -\sum_{z=1, z \neq m}^{l}\left[c_{m z}^{*}\left(p_{m}(t)\right)+\Delta c_{m z}(t)\right] f_{z}\left(p_{z}(t)\right) \\
& -\sum_{z=1}^{l}\left[d_{m z}^{*}\left(p_{m}(t)\right)+\Delta d_{m z}(t)\right] f_{z}\left(p_{z}\left(t-\tau_{m z}(t)\right)\right)-I_{m}, m=1,2, \ldots, l,
\end{aligned}
$$


Combining with $-a_{m}\left(p_{m}(t)\right)>0$, systems (14) and (2) have the same number of equilibrium points. Denote $t^{(0)}=\max _{1 \leq m, z \leq l}\left\{t_{m z}^{*}\right\}$. Then for $t \in\left[t^{(0)},+\infty\right)$, we can rewrite the function $G_{m}\left(p_{m}(t)\right)$ as follows.

$$
\begin{aligned}
& G_{m}\left(p_{m}(t)\right)=-h_{m}\left(p_{m}(t)\right)=b_{m} p_{m}(t)-\left[c_{m m}^{*}\left(p_{m}(t)\right)\right. \\
& \left.+\alpha_{m m}\right] f_{m}\left(p_{m}(t)\right)-\sum_{z=1, z \neq m}^{l}\left[c_{m z}^{*}\left(p_{m}(t)\right)+\alpha_{m z}\right] f_{z}\left(p_{z}(t)\right) \\
& -\sum_{z=1}^{l}\left[d_{m z}^{*}\left(p_{m}(t)\right)+\beta_{m z}\right] f_{z}\left(p_{z}\left(t-\tau_{m z}(t)\right)\right)-I_{m},
\end{aligned}
$$

When $p_{m}(t)=Q_{m}^{j}$ or $p_{m}(t)=U_{m}^{j}$, we have

$$
\begin{aligned}
& G_{m}\left(Q_{m}^{j}\right)=-h_{m}\left(Q_{m}^{j}\right)=b_{m} Q_{m}^{j}-\left[c_{m m}^{*}\left(Q_{m}^{j}\right)+\alpha_{m m}\right] \\
& \times f_{m}\left(Q_{m}^{j}\right)-\sum_{z=1, z \neq m}^{l}\left[c_{m z}^{*}\left(Q_{m}^{j}\right)+\alpha_{m z}\right] f_{z}\left(p_{z}(t)\right) \\
& -\sum_{z=1}^{l}\left[d_{m z}^{*}\left(Q_{m}^{j}\right)+\beta_{m z}\right] f_{z}\left(p_{z}\left(t-\tau_{m z}(t)\right)\right)-I_{m} \\
& \geq b_{m} Q_{m}^{j}-\max \left\{\tilde{c}_{m m} f_{m}\left(Q_{m}^{j}\right), \bar{c}_{m m} f_{m}\left(Q_{m}^{j}\right)\right\} \\
& -\max \left\{\alpha_{m m}^{(1)} f_{m}\left(Q_{m}^{j}\right), \alpha_{m m}^{(2)} f_{m}\left(Q_{m}^{j}\right)\right\} \\
& -\Phi_{m}^{1}-\Phi_{m}^{2}-I_{m}>0,
\end{aligned}
$$

and

$$
\begin{aligned}
& G_{m}\left(U_{m}^{j}\right)=-h_{m}\left(U_{m}^{j}\right)=b_{m} U_{m}^{j}-\left[c_{m m}^{*}\left(U_{m}^{j}\right)+\alpha_{m m}\right] \\
& \times f_{m}\left(U_{m}^{j}\right)-\sum_{z=1, z \neq m}^{l}\left[c_{m z}^{*}\left(U_{m}^{j}\right)+\alpha_{m z}\right] f_{z}\left(p_{z}(t)\right) \\
& -\sum_{z=1}^{l}\left[d_{m z}^{*}\left(U_{m}^{j}\right)+\beta_{m z}\right] f_{z}\left(p_{z}\left(t-\tau_{m z}(t)\right)\right)-I_{m} \\
& \leq b_{m} U_{m}^{j}-\min \left\{\tilde{c}_{m m} f_{m}\left(U_{m}^{j}\right), \bar{c}_{m m} f_{m}\left(U_{m}^{j}\right)\right\} \\
& -\min \left\{\alpha_{m m}^{(1)} f_{m}\left(U_{m}^{j}\right), \alpha_{m m}^{(2)} f_{m}\left(U_{m}^{j}\right)\right\} \\
& -\Gamma_{m}^{1}-\Gamma_{m}^{2}-I_{m}<0,
\end{aligned}
$$

$j=0,1, \ldots, w$. Using the continuity of $G_{m}(z)$, there exists at least a point $\tilde{p}_{m} \in\left[Q_{m}^{j}, U_{m}^{j}\right]$, such that $G_{m}\left(\tilde{p}_{m}\right)=0$, where $j=0,1, \ldots, w$. As the subset $\Theta_{2}$ consists of $(w+1)^{l}$ parts, we can obtain that there exist at least $(w+1)^{l}$ equilibrium points for the perturbed MCGNN system (2) in $\mathfrak{R}^{l}$.

2) If $p_{m}(t)$ is the solution of the perturbed MCGNN system (2) with the corresponding initial condition $\Upsilon_{m}^{(1)}(s) \in$ $\bar{\Theta}_{2}, s \in[-\tau, 0]$, we can assert $p_{m}(t) \in \bar{\Theta}_{2}$ for any $t \geq 0$. If it is false, there exists $t_{0}>0$ so that $p_{m}\left(t_{0}\right)=Q_{m}^{j},\left.\dot{p}_{m}(t)\right|_{t=t_{0}}<$ 0 or $p_{m}\left(t_{0}\right)=U_{m}^{j},\left.\dot{p}_{m}(t)\right|_{t=t_{0}}>0, j \in\{0,1, \ldots, w\}$. However, when $p_{m}\left(t_{0}\right)=Q_{m}^{j}$, we have $\left.\dot{p}_{m}(t)\right|_{t=t_{0}} \geq-a_{m}\left(Q_{m}^{j}\right)\left(-\mathrm{K}_{1}\right)>$ 0. Similarly, we can obtain $\left.\dot{p}_{m}(t)\right|_{t=t_{0}} \leq-a_{m}\left(U_{m}^{j}\right)\left(-\mathrm{K}_{2}\right)<0$ when $p_{m}\left(t_{0}\right)=U_{m}^{j}$. So, it is contradictory. Thus, $\Theta_{2}$ is a positive invariant set.

3) Denote a function as follows

$$
\kappa_{k}(v)=-\left|b_{k}\right| a_{k}^{(4)}-v+\sum_{z=1}^{l}\left(\hat{c}_{k z}+\hat{\alpha}_{k z}\right) L_{z} a_{z}^{(3)}+e^{v \tau} \sum_{z=1}^{l}\left(\hat{d}_{k z}+\hat{\beta}_{k z}\right) L_{z} a_{z}^{(3)},
$$

it is clear that $\kappa_{k}(0)>0$, and there exists a sufficiently small positive constant $\beta$, such that $\kappa_{k}(\beta)>0, k=1,2, \ldots, l$.

Let $p(t)=\left(p_{1}(t), p_{2}(t), \ldots, p_{l}(t)\right)^{T}$ be the solution of the disturbed MCGNN system (2) with initial condition $\Upsilon^{(1)}=\left(\Upsilon_{1}^{(1)}(s), \Upsilon_{2}^{(1)}(s), \ldots, \Upsilon_{l}^{(1)}(s)\right)^{T}$ and $g_{m}(t)=q_{m}(t)-q_{m}^{\#}$, where $q_{m}(t)=r_{m}\left(p_{m}(t)\right), q_{m}^{\#}=r_{m}\left(p_{m}^{\#}\right)$.

From Lemma 1, we can obtain

$$
\begin{aligned}
& \left|c o\left[c_{m z}\left(r_{m}^{-1}\left(q_{m}(t)\right)\right)\right] f_{z}\left(r_{z}^{-1}\left(q_{z}(t)\right)\right)-c o\left[c_{m z}\left(r_{m}^{-1}\left(q_{m}^{\#}\right)\right)\right] f_{z}\left(r_{z}^{-1}\left(q_{z}^{\#}\right)\right)\right| \\
& \leq \hat{c}_{m z} L_{z}^{(2)}\left|r_{z}^{-1}\left(q_{z}(t)\right)-r_{z}^{-1}\left(q_{z}^{\#}\right)\right| \leq-\hat{c}_{m z} L_{z}^{(2)} a_{m}^{(3)}\left|g_{m}(t)\right|,
\end{aligned}
$$

and

$$
\begin{aligned}
& \left|c o\left[d_{m z}\left(r_{m}^{-1}\left(q_{m}(t)\right)\right)\right] f_{z}\left(r_{m}^{-1}\left(q_{m}\left(t-\tau_{m z}(t)\right)\right)\right)-c o\left[c_{m z}\left(r_{m}^{-1}\left(q_{m}^{\#}\right)\right)\right] f_{z}\left(r_{z}^{-1}\left(q_{z}^{\#}\right)\right)\right| \\
& \left.\leq \hat{d}_{m z} L_{z}^{(2)}\left|r_{m}^{-1}\left(q_{m}\left(t-\tau_{m z}(t)\right)\right)-r_{z}^{-1}\left(q_{z}^{\#}\right)\right| \leq-\hat{d}_{m z} L_{z}^{(2)} a_{m}^{(3)} \mid g_{m}\left(t-\tau_{m z}(t)\right)\right) \mid .
\end{aligned}
$$


Let $x_{m}(t)=e^{\beta t}\left|g_{m}(t)\right|$ and $\ell=\max _{1 \leq m \leq l}\left\{\left|q_{m}(0)-q_{m}^{\#}\right|\right\}$. We can have $x_{m}(0) \leq \ell$ for any $m=1,2, \ldots, l$. Then we will prove the following inequality

$$
x_{m}(t) \leq \ell, t>0, m=1,2, \ldots, l
$$

Supposing (15) is invalid, then we can find a $k \in\{1,2, \ldots, l\}$ and $t_{1}$ for the first time $x_{k}\left(t_{1}\right)=\ell, \dot{x}_{k}\left(t_{1}\right)>0, x_{k}(t) \leq \ell$, $t \in\left[0, t_{1}\right) ; x_{j}(t) \leq \ell, t \in\left[0, t_{1}\right], j=1,2, \ldots, l, j \neq k$.

$$
\left.\frac{d x_{k}(t)}{d t}\right|_{t=t_{1}}=\beta x_{k}\left(t_{1}\right)+\left.e^{\beta t_{1}} \operatorname{sign}\left(g_{k}\left(t_{1}\right)\right) \frac{d g_{k}(t)}{d t}\right|_{t=t_{1}}
$$

Because $\frac{d}{d q_{m}(t)} r_{m}^{-1}\left(q_{m}(t)\right)=a_{m}\left(p_{m}(t)\right)<0$, we have

$$
\begin{aligned}
& -\operatorname{sign}\left(g_{m}\left(t_{1}\right)\right)\left[b_{m} r_{m}^{-1}\left(q_{m}\left(t_{1}\right)\right)-b_{m} r_{m}^{-1}\left(q_{m}^{\#}\right)\right] \\
& =b_{m} \operatorname{sign}\left(r_{m}^{-1}\left(q_{m}\left(t_{1}\right)\right)-r_{m}^{-1}\left(q_{m}^{\#}\right)\right)\left[r_{m}^{-1}\left(q_{m}\left(t_{1}\right)\right)-r_{m}^{-1}\left(q_{m}^{\#}\right)\right] \\
& \left.=-\left|b_{m}\right| \mid r_{m}^{-1}\left(q_{m}\left(t_{1}\right)\right)\right)-r_{m}^{-1}\left(q_{m}^{\#}\right) \mid \\
& \leq\left|b_{m}\right| a_{m}^{(4)}\left|q_{m}\left(t_{1}\right)-q_{m}^{\#}\right| .
\end{aligned}
$$

Then,

$$
\begin{aligned}
& \left.\frac{d x_{k}(t)}{d t}\right|_{t=t_{1}} \leq \beta x_{k}\left(t_{1}\right)+e^{\beta t_{1}}\left\{-b_{k} \operatorname{sign}\left(g_{k}\left(t_{1}\right)\right)\right. \\
& \left(r_{k}^{-1}\left(q_{k}\left(t_{1}\right)\right)-r_{k}^{-1}\left(q_{k}^{\#}\right)\right)-\sum_{z=1}^{l} \hat{c}_{k z} L_{z}^{(2)} a_{z}^{(3)}\left|g_{z}\left(t_{1}\right)\right| \\
& -\sum_{z=1}^{l} \hat{\alpha}_{k z} L_{z}^{(2)} a_{z}^{(3)}\left|g_{z}\left(t_{1}\right)\right|-\sum_{z=1}^{l} \hat{d}_{k z} L_{z}^{(2)} a_{z}^{(3)} \\
& \left.\times\left|g_{z}\left(t_{1}-\tau_{k z}\left(t_{1}\right)\right)\right|-\sum_{z=1}^{l} \hat{\beta}_{k z} L_{z}^{(2)} a_{z}^{(3)}\left|g_{z}\left(t_{1}-\tau_{k z}\left(t_{1}\right)\right)\right|\right\} \\
& \leq\left(\beta+\left|b_{k}\right| a_{k}^{(4)}-\sum_{z=1}^{l}\left(\hat{c}_{k z}+\hat{\alpha}_{k z}\right) L_{z}^{(2)} a_{z}^{(3)}\right) x_{k}\left(t_{1}\right) \\
& -e^{\beta \tau} \sum_{z=1}^{l}\left(\hat{d}_{k z}+\hat{\beta}_{k z}\right) L_{z}^{(2)} a_{z}^{(3)} x_{k}\left(t_{1}-\tau_{k z}\left(t_{1}\right)\right) \\
& \leq\left(\beta+\left|b_{k}\right| a_{k}^{(4)}-\sum_{z=1}^{l}\left(\hat{c}_{k z}+\hat{\alpha}_{k z}\right) L_{z}^{(2)} a_{z}^{(3)}\right. \\
& \left.-e^{\beta \tau} \sum_{z=1}^{l}\left(\hat{d}_{k z}+\hat{\beta}_{k z}\right) L_{z}^{(2)} a_{z}^{(3)}\right) \\
& \times \max _{1 \leq m \leq l}\left(\left|q_{m}(0)-q_{m}^{\#}\right|\right\}<0
\end{aligned}
$$

So,

$$
x_{m}(t)=e^{\beta t}\left|g_{m}(t)\right| \leq\left|q_{m}(0)-q_{m}^{\#}\right|,
$$

that is

$$
-\frac{e^{\beta t}}{a_{m}^{(3)}}\left|p_{m}(t)-p_{m}^{\#}\right| \leq-\frac{1}{a_{m}^{(4)}}\left|p_{m}(0)-p_{m}^{\#}\right|
$$

Accordingly, we obtain

$$
\left|p_{m}(t)-p_{m}^{\#}\right| \leq \frac{a_{m}^{(3)}}{a_{m}^{(4)}}\left|p_{m}(0)-p_{m}^{\#}\right| e^{-\beta t}
$$

Thus,

$$
\left\|p(t)-p^{\#}\right\| \leq \alpha\left\|\Upsilon^{(1)}-p^{\#}\right\| e^{-\beta t}
$$

where $\alpha=\max _{1 \leq m \leq l}\left\{\frac{a_{m}^{(3)}}{a_{m}^{(4)}}\right\}$.

Therefore, the equilibrium point $p^{\#}=\left(p_{1}^{\#}, p_{2}^{\#}, \ldots, p_{l}^{\#}\right)^{T}$ of MCGNN (2) is exponentially stable. Further, the perturbed MCGNN system (2) can have at least $(w+1)^{l}$ exponentially stable equilibrium points in $\mathfrak{R}^{l}$. The proof is finished. 
If activation function is piecewise linear as in (10), we can have the following corollary.

Corollary 2: The perturbed MCGNN system (2) has at least $(w+1)^{l}$ exponentially stable equilibrium points in $\mathfrak{R}^{l}$ and $\Theta_{2}$ is a positive invariant set, if $f_{m}\left( \pm \chi_{m}\right)=0$, assumptions $2,4,5$ and the conditions (6), (7), and

$$
-\left|b_{m}\right| a_{m}^{(4)}+\sum_{z=1}^{l}\left(\hat{c}_{m z}+\hat{\alpha}_{m z}\right) l_{z}^{\max } a_{z}^{(3)}+\sum_{z=1}^{l}\left(\hat{d}_{m z}+\hat{\beta}_{m z}\right) l_{z}^{\max } a_{z}^{(3)}>0
$$

hold, where $l_{z}^{\max }=\max \left\{\gamma_{z}^{0}, \gamma_{z}^{1}, \ldots, \gamma_{z}^{w}\right\}$.

Remark 5: In the past few years, the multistability problem of MNNs has been widely investigated. However, the ranges in which stable equilibrium points of MNNs locate are limited to the odd-sequence regions (i.e. oddsequence positive invariant regions) $[27-32,34]$. In this paper, we investigate that these stable equilibrium points can locate in the even-sequence regions (i.e. even-sequence positive invariant regions). Take a MCGNN system with $p(t)=\left(p_{1}(t), p_{2}(t)\right)^{T}$ as an example. The existing results showed that the ranges in which stable equilibrium points of MNNs locate are the odd-sequence rectangular regions marked with yellow color in Fig. 1. In this paper, we have found that these stable equilibrium points can locate in the even-sequence rectangular regions marked with red color in Fig. 1. More specifically, the odd-sequence rectangular regions and even-sequence rectangular regions shown in Fig. 1 are represented as $\Theta_{1}$ and $\Theta_{2}$, respectively.

$$
\begin{aligned}
\Theta_{1}= & \left\{\prod_{m=1}^{2}\left(-\infty, Q_{m}^{0}\right]^{\varepsilon_{m}^{1}} \times\left(Q_{m}^{0}, U_{m}^{0}\right)^{0} \times\left[U_{m}^{0}, Q_{m}^{1}\right]^{\varepsilon_{m}^{2}}\right. \\
& \times \cdots \times\left(Q_{m}^{w}, U_{m}^{w}\right)^{0} \times\left[U_{m}^{w},+\infty\right)^{\varepsilon_{m}^{w+2}} \mid \\
& \left(\varepsilon_{m}^{1}, \varepsilon_{m}^{2}, \ldots, \varepsilon_{m}^{w+2}\right)=(1,0, \ldots, 0) \text { or } \\
& (0,1, \ldots, 0) \text { or } \ldots \text { or }(0,0, \ldots, 1)\},
\end{aligned}
$$

and

$$
\begin{aligned}
\Theta_{2}= & \left\{\prod_{m=1}^{2}\left(-\infty, Q_{m}^{0}\right]^{0} \times\left(Q_{m}^{0}, U_{m}^{0}\right)^{\varepsilon_{m}^{1}} \times\left[U_{m}^{0}, Q_{m}^{1}\right]^{0}\right. \\
& \times \cdots \times\left(Q_{m}^{w}, U_{m}^{w}\right)_{m}^{\varepsilon_{m}^{w+1}} \times\left[U_{m}^{w},+\infty\right)^{0} \mid \\
& \left(\varepsilon_{m}^{1}, \varepsilon_{m}^{2}, \ldots, \varepsilon_{m}^{w+1}\right)=(1,0, \ldots, 0) \text { or } \\
& (0,1, \ldots, 0) \text { or } \ldots \text { or }(0,0, \ldots, 1)\} .
\end{aligned}
$$

Therefore, the obtained result is an important supplement to the previous research results, and extends the existing results $[27-32,34]$.

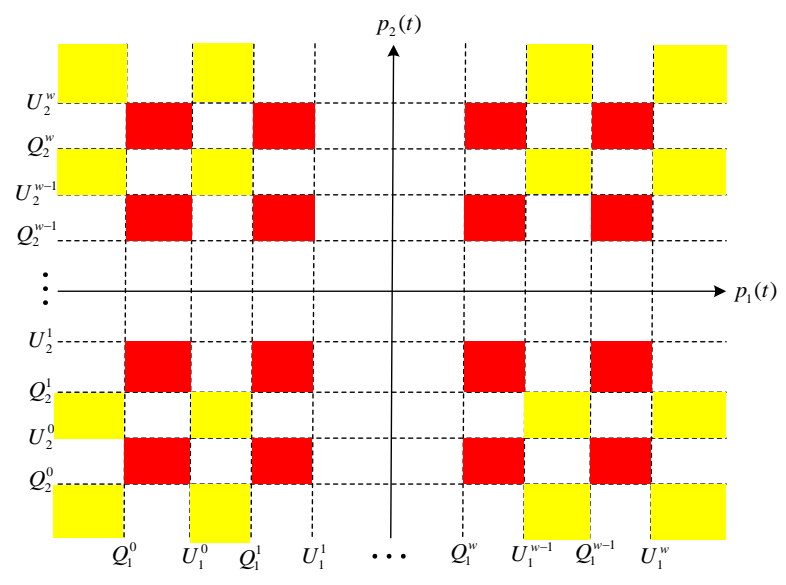

Figure 1: The rectangular regions in which stable equilibrium points of $p(t)=\left(p_{1}(t), p_{2}(t)\right)^{T}$ locate. The odd-sequence rectangular regions are marked with yellow color, and the even-sequence rectangular regions are marked with red color.

Remark 6: In this paper, some comparisons with the previous works [34-37] are given in Table 1. We make comparisons from four aspects: activation function; the number of stable equilibrium points; regions of stable equilib- 
Table 1: Some comparisons with the previous works [34-37].

\begin{tabular}{ccccc}
\hline Paper & AF & Number & Region & Anti-interference \\
\hline$[34]$ & piecewise linear & $2^{l}$ & odd-sequence & No \\
{$[35]$} & piecewise linear & $3^{l}$ & odd-sequence & No \\
{$[36]$} & piecewise linear & $3^{l}$ & odd-sequence & No \\
{$[37]$} & piecewise nonlinear & $3^{4 l}$ & odd-sequence & No \\
This paper & piecewise nonlinear & $(w+2)^{l}$ or $(w+1)^{l}$ & odd-sequence or even-sequence & Yes \\
\hline
\end{tabular}

rium points and anti-interference performance. For convenience, activation function, the number of stable equilibrium points, regions of stable equilibrium points and anti-interference performance are simplified as AF, Number, Region and Anti-interference, respectively. Then we can obtain the following advantages of multistability of MCGNNs with stochastic parameter perturbations: 1) The conditions for activation function of this paper are more flexible; 2) The MCGNN system possesses more equilibrium points; 3) The stable equilibrium points of MCGNNs can be flexibly located in the odd-sequence or the even-sequence regions; 4) The obtained results are robust against perturbations.

Remark 7: Currently, some works on multistability of NNs including MCGNNs without the effects of parameter perturbations have been investigated [23-40]. However, some unpredictable factors may lead to parameter perturbations randomly changing along with time. What is more, parameter perturbations of NNs may lead to instability and some unpredictable influence for the systems. Therefore, the aim of this paper is to derive some sufficient conditions to guarantee the exponential multistability of MCGNNs under stochastic parameter perturbations.

Remark 8: There exist three main technical difficulties to investigate the exponential multistability of MCGNNs with stochastic parameter perturbations, which are presented as follows.

1. It is complicated and inconvenient to analyze MCGNN system (3) due to the amplification function $a_{m}\left(p_{m}(t)\right)$. Moreover, it is difficult to simplify system (3) into system (5). This paper uses derivative theorem of inverse function to eliminate the amplification function $a_{m}\left(p_{m}(t)\right)$, such that MCGNN system (3) can be simplified into system (5).

2. It is very hard to give sufficient conditions of theorems and corollaries to achieve the exponential multistability of MCGNNs. Firstly, fixed point theorem is used to acquire multiple equilibrium points, for example, the steps 1) and 2) of the proof of the Theorem 1. Then, set-valued maps theory and differential inequality method are applied to prove that these equilibrium points are exponentially stable, for example, the step 3) of the proof of Theorem 1.

3. Stochastic parameter perturbations easily lead to instability of system and increase difficulty in giving sufficient conditions for exponential multistability of MCGNNs. Therefore, we present assumption 4 to solve the difficulty in Theorem 1 and Theorem 2.

\section{Numerical simulation}

In this section, we provide two examples to verify the validity of the obtained results.

Example 1. Consider a MCGNN system with stochastic parameter perturbations as

$$
\begin{aligned}
& \dot{p}_{m}(t)=a_{m}\left(p_{m}(t)\right)\left\{-b_{m} p_{m}(t)+\sum_{z=1}^{2}\left[c_{m z}\left(p_{m}(t)\right)+\Delta c_{m z}(t)\right] f_{z}\left(p_{z}(t)\right)\right. \\
& \left.+\sum_{z=1}^{2}\left[d_{m z}\left(p_{m}(t)\right)+\Delta d_{m z}(t)\right] f_{z}\left(p_{z}\left(t-\tau_{m z}(t)\right)\right)+I_{m}\right\}, m=1,2,
\end{aligned}
$$

where $a_{1}\left(p_{1}(t)\right)=1+0.03 \tanh \left(p_{1}(t)\right), a_{2}\left(p_{2}(t)\right)=1.01+0.01 \tanh \left(p_{2}(t)\right)$,

$$
\begin{aligned}
& c_{11}\left(p_{1}(t)\right)= \begin{cases}4.42, & \left|p_{1}(t)\right| \leq 3.6, \\
4.58, & \left|p_{1}(t)\right|>3.6,\end{cases} \\
& c_{22}\left(p_{2}(t)\right)= \begin{cases}4.65, & \left|p_{2}(t)\right| \leq 3.6, \\
3.96, & \left|p_{2}(t)\right|>3.6,\end{cases} \\
& d_{11}\left(p_{1}(t)\right)= \begin{cases}0.02, & \left|p_{1}(t)\right| \leq 3.6, \\
0.15, & \left|p_{1}(t)\right|>3.6,\end{cases}
\end{aligned}
$$




$$
\begin{gathered}
d_{22}\left(p_{2}(t)\right)=\left\{\begin{array}{cc}
0.16, & \left|p_{2}(t)\right| \leq 3.6, \\
0.21, & \left|p_{2}(t)\right|>3.6,
\end{array}\right. \\
c_{m z}=0, d_{m z}=0, m \neq z ; \text { input } I_{1}=I_{2}=2, \tau_{m z}(t)=0.1, \Delta c_{12}\left(p_{1}(t)\right)=\Delta c_{21}\left(p_{2}(t)\right)=0, \\
\Delta c_{11}(t)=\left\{\begin{array}{cc}
-0.1+0.02 t, & t \in[0,8) ; \\
0.06, & t \in[8,+\infty) ;
\end{array}\right. \\
\Delta c_{22}\left(p_{2}(t)\right)=\left\{\begin{array}{cc}
0.04+0.015 \tanh (t-6.6), & t \in[0,6.6) ; \\
0.04, & t \in[6.6,+\infty) ;
\end{array}\right.
\end{gathered}
$$

$\Delta d_{m z}\left(p_{m}(t)\right)=0, b_{m}=2, m, z=1,2$.

The activation function $f_{z}(r), z=1,2$, is denoted by

$$
f_{z}(r)= \begin{cases}-4+0.02 \sin (r+4), & r \in(-\infty,-4], \\ 10(r+3.6), & r \in(-4,-3.6), \\ -\frac{2}{7} r-\frac{36}{35}, & r \in[-3.6,3.4], \\ 10(r-3.6), & r \in(3.4,4), \\ 4, & r \in[4,+\infty) .\end{cases}
$$

We can find $f_{z}( \pm 3.6)=0$, and $L_{z}^{(1)}=\frac{2}{7}, z=1,2$. Through simple calculation, we can obtain that the conditions of Theorem 1 are satisfied. Therefore, there exist at least 9 exponentially stable equilibrium points for the perturbed MCGNN (17) in the $\Theta_{1}^{*}$ and $\Theta_{1}^{*}$ is a positive invariant set, where

$$
\begin{aligned}
\Theta_{1}^{*}= & \left\{\prod_{m=1}^{2}(-\infty,-4]^{\varepsilon_{m}^{1}} \times(-4,-3.6)^{0} \times[-3.6,3.4]^{\varepsilon_{m}^{2}} \times(3.4,4)^{0} \times[4,+\infty)^{\varepsilon_{m}^{3}} \mid\right. \\
& \left.\left(\varepsilon_{m}^{1}, \varepsilon_{m}^{2}, \varepsilon_{m}^{3}\right)=(1,0,0) \operatorname{or}(0,1,0) \operatorname{or}(0,0,1)\right\} .
\end{aligned}
$$

The state trajectories of $p_{1}(t)$ and $p_{2}(t)$ of the perturbed MCGNN (17) are traced with 270 initial values located in the $\Theta_{1}^{*}$, and the simulation results are shown in Fig. 2. From Fig. 2, there exist at least 9 exponentially stable equilibrium points in the $\Theta_{1}^{*}$, that is odd-sequence rectangular regions marked with yellow color. Figs. 3-5 shows the trajectories of $p_{1}(t)$ and $p_{2}(t)$ along with time $t$.

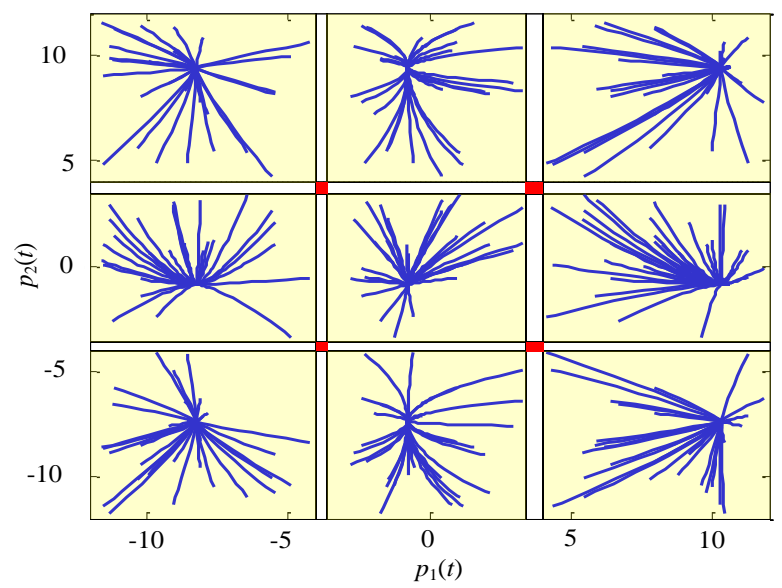

Figure 2: The trajectories of $p_{1}(t)$ and $p_{2}(t)$ with positive $a_{m}\left(p_{m}(t)\right)$. There exist at least 9 exponentially stable equilibrium points in the oddsequence rectangular regions marked with yellow color.

Example 2. Consider another MCGNN system with stochastic parameter perturbations as

$$
\begin{aligned}
& \dot{p}_{m}(t)=a_{m}\left(p_{m}(t)\right)\left\{-b_{m}\left(p_{m}(t)\right)+\sum_{z=1}^{2}\left[c_{m z}\left(p_{m}(t)\right)+\Delta c_{m z}(t)\right] f_{z}\left(p_{z}(t)\right)\right. \\
& \left.+\sum_{z=1}^{2}\left[d_{m z}\left(p_{m}(t)\right)+\Delta d_{m z}(t)\right] f_{z}\left(p_{z}\left(t-\tau_{m z}(t)\right)\right)+I_{m}\right\}, m=1,2,
\end{aligned}
$$




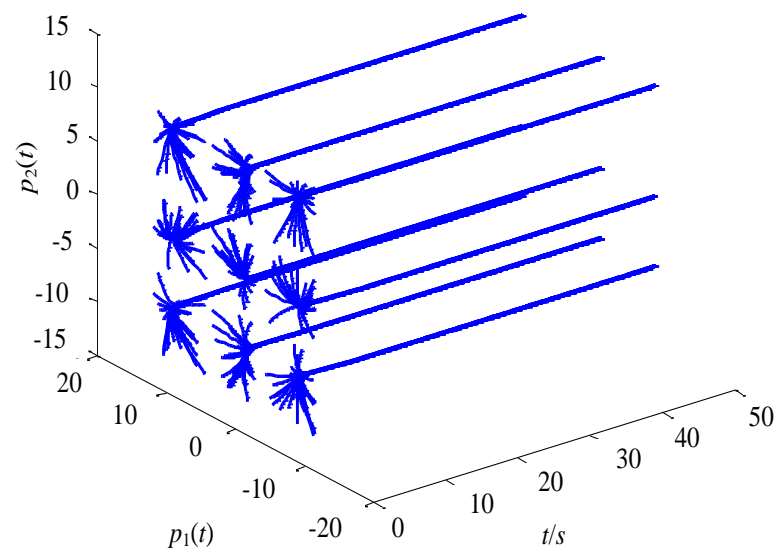

Figure 3: The trajectories of $p_{1}(t)$ and $p_{2}(t)$ along with time $t$ in Example 1. There exist at least 9 trajectories of $p_{1}(t)$ and $p_{2}(t)$ that converge to 9 stable equilibrium points along with time $t$.

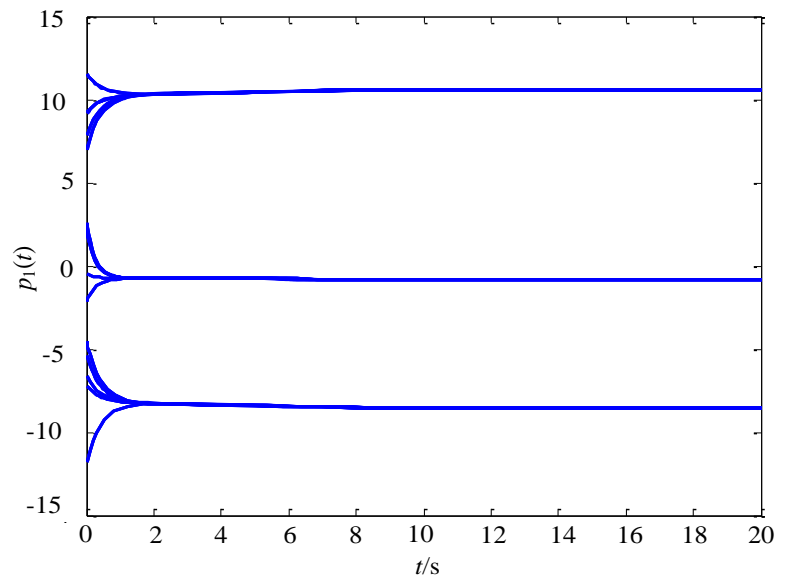

Figure 4: The trajectories of $p_{1}(t)$ along with time $t$ in Example 1. There exist at least 3 trajectories of $p_{1}(t)$ that converge to 3 stable equilibrium states along with time $t$.

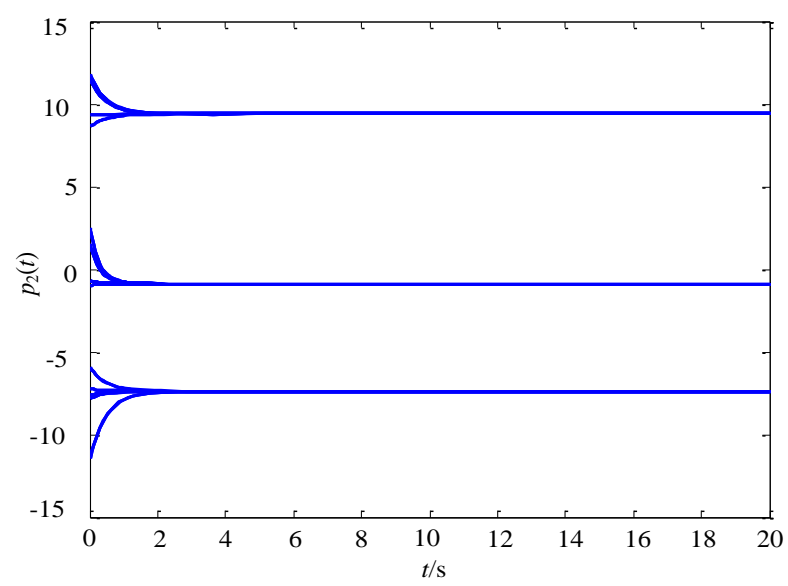

Figure 5: The trajectories of $p_{2}(t)$ along with time $t$ in Example 1. There exist at least 3 trajectories of $p_{2}(t)$ that converge to 3 stable equilibrium states along with time $t$. 
where $a_{1}\left(p_{1}(t)\right)=-1+0.03 \tanh \left(p_{1}(t)\right), a_{2}\left(p_{2}(t)\right)=-1.02-0.01 \tanh \left(p_{2}(t)\right)$,

$$
\begin{aligned}
& c_{11}\left(p_{1}(t)\right)= \begin{cases}9.85, & \left|p_{1}(t)\right| \leq 0.7, \\
10.2, & \left|p_{1}(t)\right|>0.7,\end{cases} \\
& c_{22}\left(p_{2}(t)\right)= \begin{cases}9.96, & \left|p_{2}(t)\right| \leq 0.7, \\
10.12, & \left|p_{2}(t)\right|>0.7,\end{cases} \\
& d_{11}\left(p_{1}(t)\right)= \begin{cases}0.01, & \left|p_{1}(t)\right| \leq 0.7, \\
0.05, & \left|p_{1}(t)\right|>0.7,\end{cases} \\
& d_{22}\left(p_{2}(t)\right)= \begin{cases}0.09, & \left|p_{2}(t)\right| \leq 0.7, \\
0.18, & \left|p_{2}(t)\right|>0.7,\end{cases}
\end{aligned}
$$

input $I_{1}=I_{2}=0.5, \tau_{m z}(t)=0.1, \Delta c_{12}\left(p_{1}(t)\right)=\Delta c_{21}\left(p_{2}(t)\right)=0$,

$$
\begin{gathered}
\Delta c_{11}(t)=\left\{\begin{array}{cc}
0.05+0.02 \sin (t-6), & t \in[0,6) ; \\
0.05, & t \in[6,+\infty) ;
\end{array}\right. \\
\Delta c_{22}\left(p_{2}(t)\right)=\left\{\begin{array}{cc}
0.02+0.03 \exp (t-5.8), & t \in[0,5.8) ; \\
0.05, & t \in[5.8,+\infty) ;
\end{array}\right.
\end{gathered}
$$

$\Delta d_{m z}\left(p_{m}(t)\right)=0, b_{m}=-10, m, z=1,2$. The activation function $f_{z}(r), z=1,2$, is denoted by

$$
f_{z}(r)= \begin{cases}-0.1+0.01 \sin (r+1), & r \in(-\infty,-1], \\ \frac{1}{3} r+\frac{0.7}{3}, & r \in(-1,-0.1), \\ -9 r-0.7, & r \in[-0.1,-0.05], \\ \frac{1}{3} r-\frac{0.7}{3}, & r \in(-0.05,1), \\ 0.1+0.01 \tanh (r-1), & r \in[1,+\infty) .\end{cases}
$$

We can find $f_{z}( \pm 0.7)=0$, and $L_{z}^{(2)}=\frac{1}{3}, z=1,2$. Then the conditions of Theorem 2 are satisfied by simple calculation. Therefore, there exist at least 4 exponentially stable equilibrium points for the perturbed MCGNN (18) in the $\Theta_{2}^{*}$ and $\Theta_{2}^{*}$ is a positive invariant set, where

$$
\begin{aligned}
\Theta_{2}^{*} & =\left\{\prod_{m=1}^{2}(-\infty,-1]^{0} \times(-1,-0.1)^{\varepsilon_{m}^{1}} \times[-0.1,-0.05]^{0}\right. \\
& \left.\times(-0.05,1)^{\varepsilon_{m}^{2}} \times[1,+\infty)^{0} \mid\left(\varepsilon_{m}^{1}, \varepsilon_{m}^{2}\right)=(1,0) \operatorname{or}(0,1)\right\} .
\end{aligned}
$$

The state trajectories of $p_{1}(t)$ and $p_{2}(t)$ of the perturbed MCGNN (18) with 120 initial values located in the $\Theta_{2}^{*}$ are shown in Fig. 6. From Fig. 6, there exist at least 4 exponentially stable equilibrium points in the $\Theta_{2}^{*}$, that is even-sequence rectangular regions marked with red color. Figs. 7-9 show the trajectories of $p_{1}(t)$ and $p_{2}(t)$ along with time $t$.

\section{Conclusions}

This paper has investigated the multistability issue of MCGNNs with parameter perturbations. Since some unpredictable factors of environment may result in stochastic parameter perturbations for MCGNNs, we have considered the multistability of MCGNNs with stochastic parameter perturbations. We have derived some sufficient conditions to achieve the exponential multistability of MCGNNs under the stochastic parameter perturbations. It has been shown in this paper that the stable equilibrium points of system can be flexibly located in the odd-sequence or the even-sequence regions. Therefore, the obtained result has extended the existing results in [23-40].

There usually exist some perturbations or disturbances in the circuit implementation of memristive neural networks. In this paper, multistability of MCGNN under stochastic parameter perturbations has been addressed. Actually, in the future works, it is very challenge and meaningful to investigate stability or multistability of memristive neural networks under some disturbances such as impulsive disturbance, uncertain disturbance. In addition, it would also be a very interesting topic to study finite-time stability (or finite-time multistability) of MCGNNs with stochastic parameter perturbations in the future. 


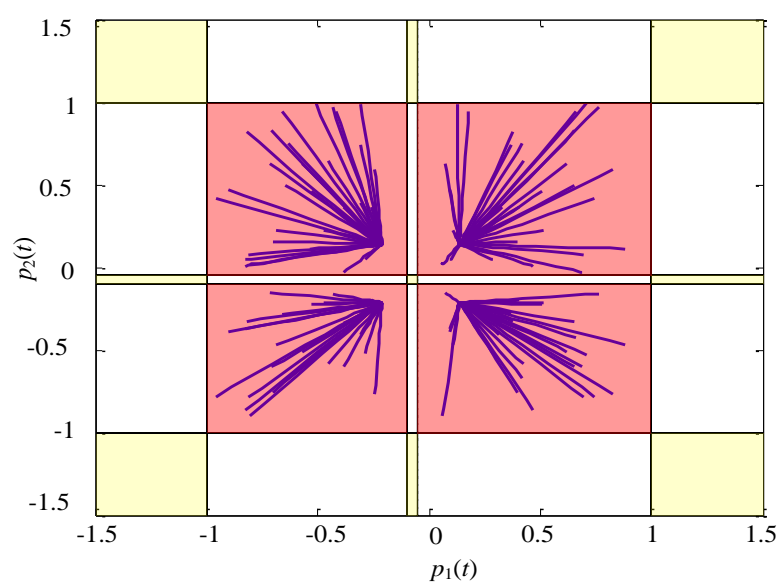

Figure 6: The trajectories of $p_{1}(t)$ and $p_{2}(t)$ with negative $a_{m}\left(p_{m}(t)\right)$. There exist at least 4 exponentially stable equilibrium points in the evensequence rectangular regions marked with red color.

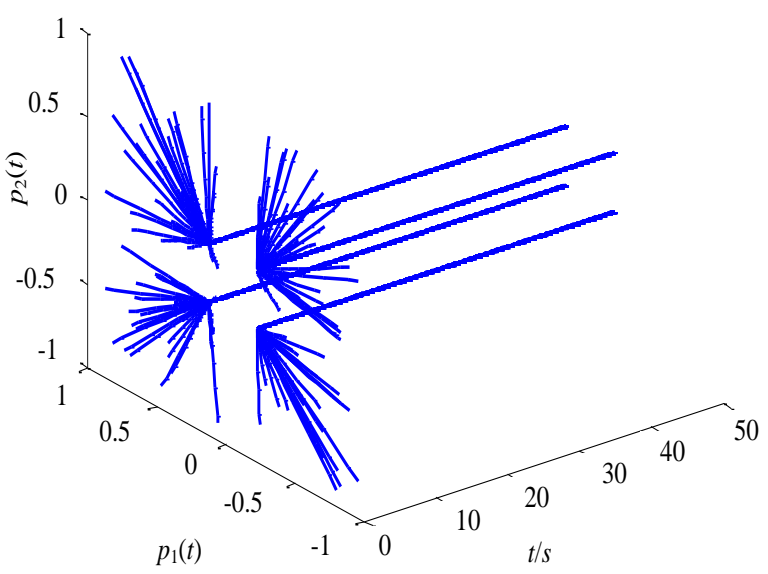

Figure 7: The trajectories of $p_{1}(t)$ and $p_{2}(t)$ along with time $t$ in Example 2. There exist at least 4 trajectories of $p_{1}(t)$ and $p_{2}(t)$ that converge to 4 stable equilibrium points along with time $t$.

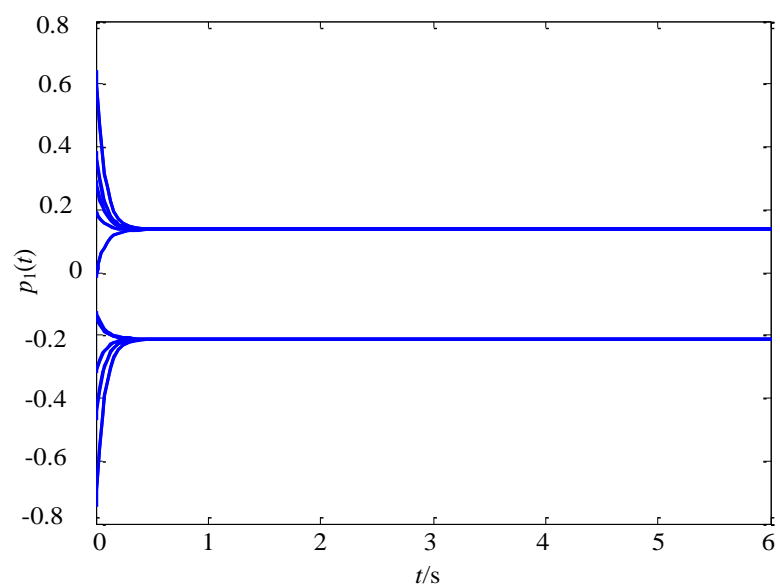

Figure 8: The trajectories of $p_{1}(t)$ along with time $t$ in Example 2. There exist at least 2 trajectories of $p_{1}(t)$ that converge to 2 stable equilibrium states along with time $t$. 


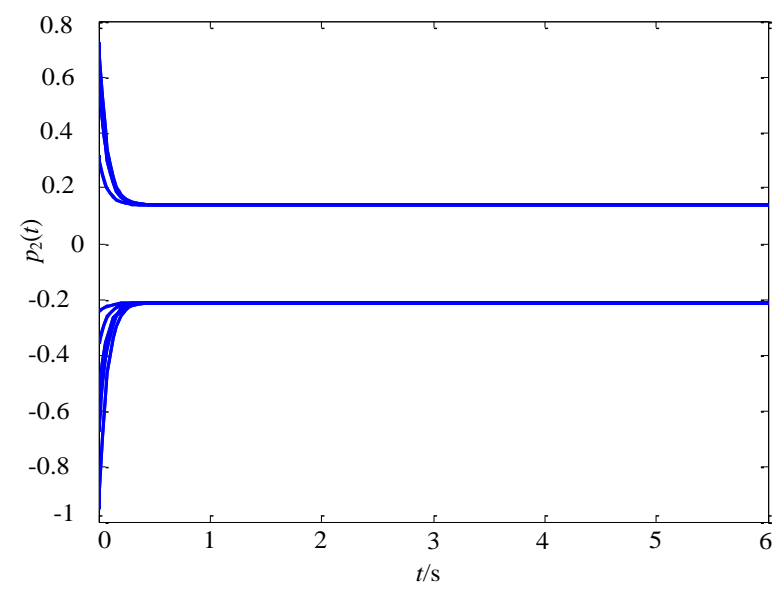

Figure 9: The trajectories of $p_{2}(t)$ along with time $t$ in Example 2. There exist at least 2 trajectories of $p_{2}(t)$ that converge to 2 stable equilibrium states along with time $t$

\section{Acknowledgments}

This work was jointly supported by the National Natural Science Foundation of China under grant no. 61971185, the Major Research Plan of the National Natural Science Foundation of China under grant no. 91964108 and the Open Fund Project of Key Laboratory in Hunan Universities under grant no. 18K010.

\section{References}

[1] J.G. Chen, K.L. Li, K. Bilal, X. Zhou, K.Q. Li, P.S. Yu, A bi-layered parallel training architecture for large-scale convolutional neural networks, IEEE Trans. Parallel Distrib. Syst. 30 (2019) 965-976.

[2] P. Melnyk, Z.Q. You, K.Q. Li, A high-performance CNN method for offline handwritten Chinese character recognition and visualization, Soft Comput. (2019). In press.

[3] C.J. Xu, M.X. Liao, P.L. Li, Y. Guo, S. Yuan, Joint influence of leakage delays and proportional delays on almost periodic solutions for FCNNs, Iran. J. Fuzzy Syst. 17 (1) (2020) 141-154.

[4] C.J. Xu, M.X. Liao, P.L. Li, Y. Guo, Q.M. Xiao, S. Yuan, Influence of multiple time delays on bifurcation of fractional-order neural networks, Appl. Math. Comput. 361 (2019) 565-582.

[5] C.J. Xu, P.L. Li, On anti-periodic solutions for neutral shunting inhibitory cellular neural networks with time-varying delays and D operator, Neurocomputing 275 (2018) 377-382.

[6] C.J. Xu, Q.M. Zhang, On anti-periodic solutions for Cohen-Grossberg shunting inhibitory neural networks with time-varying delays and impulses, Neural Comput. 26(10) (2014) 2328-2349.

[7] Z.M. Li, X.H. Chang, L. Yu, Robust quantized H-infinity filtering for discrete-time uncertain systems with packet dropouts, Appl. Math. Comput. 275 (2016) 361-371

[8] X.H. Chang, Y. Liu, M.Q. Shen, Resilient control design for lateral motion regulation of intelligent vehicle, IEEE-ASME T. Mech. 24 (6) (2019) 2488-2497.

[9] L. Liu, X.J. Xie, State feedback stabilization for stochastic feedforward nonlinear systems wi.th time-varying delay, Automatica 49 (4) (2013) 936-942.

[10] L. Liu, S. Yin, H.J. Gao, F. Alsaadi, T. Hayat, Adaptive partial-state feedback control for stochastic high-order nonlinear systems with stochastic input-to-state stable inverse dynamics, Automatica 51 (2015) 285-291.

[11] X.D. Li, J.D. Cao, M. Perc, Switching laws design for stability of finite and infinite delayed switched systems with stable and unstable modes, IEEE Access 6 (2018) 6677-6691.

[12] S.H. Ding, J.H. Park, C.C. Chen, Second-order sliding mode controller design with output constraint, Automatica 112 (2020) 108704.

[13] L.D. Fang, L. Ma, S.H. Ding, D.A. Zhao, Finite-time stabilization for a class of high-order stochastic nonlinear systems with an output constraint, Appl. Math. Comput. 358 (2019) 63-79.

[14] L.L. Zhou, C.H. Wang, S.C. Du, L. Zhou, Cluster synchronization on multiple nonlinearly coupled dynamical subnetworks of complex networks with nonidentical nodes, IEEE Trans. Neural Netw. Learn. Syst. 28 (3) (2017) 570-583.

[15] C.H. Wang, L. Xiong, J.R. Sun, W. Yao, Memristor-based neural networks with weight simultaneous perturbation training, Nonlinear Dyn. 95 (2019) 2893-2906.

[16] F. Yu, L. Liu, S. Qian, L.X. Li, Y.Y. Huang, C.Q. Shi, S. Cai, X.M. Wu, S.C. Du, Q.Z. Wan, Chaos-based application of a novel multistable 5D memristive hyperchaotic system with coexisting multiple attractors, Complexity, 2020 (2020) 8034196. 
[17] L. Zhou, C.H. Wang, L.L. Zhou, A novel no-equilibrium hyperchaotic multi-wing system via introducing memristor, Int. J. Circ. Theor. Appl. 46 (2018) 84-98.

[18] H.R. Lin, C.H. Wang, Influences of electromagnetic radiation distribution on chaotic dynamics of a neural network, Appl. Math. Comput. 369 (2020) 124840-1-124840-19.

[19] H.R. Lin, C.H. Wang, Y.M. Tan, Hidden extreme multistability with hyperchaos and transient chaos in a Hopfield neural network affected by electromagnetic radiation, Nonlinear Dyn. 99 (3) (2020) 2369-2386.

[20] C.J. Xu, P.L. Li, Y.C. Pang, Exponential stability of almost periodic solutions for memristor-based neural networks with distributed leakage delays, Neural Comput. 28(12) (2016) 2726-2756.

[21] M. Itoh, L.O. Chua, Memristor cellular automata and memristor discrete-time cellular neural networks, Int. J. Bifurc. Chaos 19 (2009) 3605-3656.

[22] S.K. Duan, X.F. Hu, Z.K. Dong, L.D. Wang, P. Mazumder, Memristor-based cellular nonlinear/neural network: design, analysis, and applications, IEEE Trans. Neural Netw. Learn. Syst. 26 (2015) 1202-1213.

[23] J.D. Cao, K. Yuan, H.X. Li, Global asymptotical stability of recurrent neural networks with multiple discrete delays and distributed delays, IEEE Trans. Neural Netw. 17 (2006) 1646-1651.

[24] X.D. Li, S.J. Song, Impulsive control for existence, uniqueness, and global stability of periodic solutions of recurrent neural networks with discrete and continuously distributed delays, IEEE Trans. Neural Netw. Learn. Syst. 24 (2013) 868-877.

[25] E. Kaslik, S. Sivasundaram, Impulsive hybrid discrete-time Hopfield neural networks with delays and multistability analysis, Neural Netw. 24 (2011) 370-377.

[26] W.H. Chen, S.X. Luo, X.M. Lu, Multistability in a class of stochastic delayed Hopfield neural networks, Neural Netw. 68 (2015) $52-61$.

[27] L.L. Wang, W.L. Lu, T.P. Chen, Multistability and new attraction basins of almost-periodic solutions of delayed neural networks, IEEE Trans. Neural Netw. 20 (2009) 1581-1593.

[28] C.Y. Cheng, K.H. Lin, C.W. Shih, J.P. Tseng, Multistability for delayed neural networks via sequential contracting, IEEE Trans. Neural Netw. Learn. Syst. 26 (2015) 3109-3122.

[29] W. Yang, Y.W. Wang, Z.G. Zeng, D.F. Zheng, Multistability of discrete-time delayed Cohen-Grossberg neural networks with second-order synaptic connectivity, Neurocomputing 164 (2015) 252-261.

[30] Z.G. Zeng, T.W. Huang, W.X. Zheng, Multistability of recurrent neural networks with time-varying delays and the piecewise linear activation function, IEEE Trans. Neural Netw. 21 (2010) 1371-1377.

[31] Z.G. Zeng, W.X. Zheng, Multistability of two kinds of recurrent neural networks with activation functions symmetrical about the origin on the phase plane, IEEE Trans. Neural Netw. Learn. Syst. 24 (2013) 1749-1762.

[32] L.L. Wang, W.L. Lu, T.P. Chen, Coexistence and local stability of multiple equilibria in neural networks with piecewise linear nondecreasing activation functions, Neural Netw. 23 (2010) 189-200.

[33] Z.Y. Guo, J. Wang, Z. Yan, Global exponential dissipativity and stabilization of memristor-based recurrent neural networks with time-varying delays, Neural Netw. 48 (2013) 158-172.

[34] A.L. Wu, J.E. Zhang, Multistability of memristive neural networks with time-varying delays, Complexity, 21 (2015) 177-186.

[35] X.B. Nie, W.X. Zheng, J.D. Cao, Coexistence and local $\mu$-stability of multiple equilibrium points for memristive neural networks with nonmonotonic piecewise linear activation functions and unbounded time-varying delays, Neural Netw. 84 (2016) $172-180$.

[36] X.B. Nie, J.L. Liang, J.D. Cao, Multistability analysis of competitive neural networks with Gaussian-wavelet-type activation functions and unbounded time-varying delays, Appl. Math. Comput. 356 (2019) 449-468.

[37] M.C. Tan, Y.F. Liu, D.S. Xu, Multistability analysis of delayed quaternion-valued neural networks with nonmonotonic piecewise nonlinear activation functions, Appl. Math. Comput. 341 (2019) 229-255.

[38] W. Yao, C.H. Wang, J.D. Cao, Y.C. Sun, C. Zhou, Hybrid multisynchronization of coupled multistable memristive neural networks with time delays, Neurocomputing 363 (2019) 281-294.

[39] D. Angeli, J. E. Ferrell, E. D. Sontag, Detection of multistability, bifurcations, and hysteresis in a large class of biological positive-feedback systems, Proc. Nat. Acad. Sci. USA 101 (2004) 1822-1827.

[40] R.J. Erichsen, L.G. Brunnet, Multistability in networks of Hindmarsh-Rose neurons, Phys. Rev. E 78 (2008) 061917.

[41] X.X. Lv, X.D. Li, J.D. Cao, M. Perc, Dynamical and static multisynchronization of coupled multistable neural networks via impulsive control, IEEE Trans. Neural Netw. Learn. Syst. 29 (12) (2018) 6062-6072.

[42] J.J. Hopfield, Neurons with graded response have collective computational properties like those of two-state neurons, Proc. Natl. Acad. Sci. USA, 81 (1984) 3088-3092.

[43] M.A. Cohen, S. Grossberg, Absolute stability of global pattern formation and parallel memory storage by competitive neural networks, IEEE Trans. Syst. Man, Cybern 13 (1983) 815-826.

[44] K. Gopalsamy, Global asymptotic stability in a periodic Lotka-Volterra system, J. Austral. Math. Soc. Ser. B 27 (1985) 66-72.

[45] X.S. Yang, J.D. Cao, W.W. Yu, Exponential synchronization of memristive Cohen-Grossberg neural networks with mixed delays, Cogn. Neurodyn. 8 (2014) 239-249.

[46] M. Liu, H.J. Jiang, C. Hu, New results for exponential synchronization of memristive Cohen-Grossberg neural networks with time-varying delays, Neural Process. Lett. 49 (2019) 79-102.

[47] X.B. Nie, W.X. Zheng, J.D. Cao, Multistability of memristive Cohen-Grossberg neural networks with non-monotonic piecewise linear activation functions and time-varying delays, Neural Netw. 71 (2015) 27-36.

[48] W.G. Yang, W.W. Yu, J.D. Cao, F.E. Alsaadi, T. Hayat, Global exponential stability and lag synchronization for delayed memristive fuzzy Cohen-Grossberg BAM neural networks with impulses, Neural Netw. 98 (2018) 122-153.

[49] S.T. Qin, Q. Ma, J.Q. Feng, C. Xu, Multistability of almost periodic solution for memristive Cohen-Grossberg neural networks with mixed delays, IEEE Trans. Neural Netw. Learn. Syst. (2019). In press.

[50] Y.H. Zhou, C.D. Li, L. Chen, T.W. Huang, Global exponential stability of memristive Cohen-Grossberg neural networks with mixed delays and impulse time window, Neurocomputing 275 (2018) 2384-2391.

[51] H. Zhao, L.X. Li, H.P. Peng, J. Kurths, J.H. Xiao, Y.X. Yang, Finite-time robust synchronization of memristive neural network with pertur- 
bation, Neural Process Lett. 47 (2018) 509-533.

[52] Y.H. Li, B. Luo, D.R. Liu, Z.Y. Yang, Robust synchronization of memristive neural networks with strong mismatch characteristics via pinning control, Neurocomputing 289 (2018) 144-154.

[53] X.S. Yang, D.W.C Ho, Synchronization of delayed memristive neural networks: robust analysis approach, IEEE Trans. Cybern. 46 (2016) 3377-3387.

[54] S.F. Yang, Z.Y. Guo, J. Wang, Robust synchronization of multiple memristive neural networks with uncertain parameters via nonlinear coupling, IEEE Trans. Syst. Man, Cybern., Syst. 45 (2015) 1077-1086.

[55] J.P. Aubin, A. Cellina, Differential inclusions. Berlin, Germany: Springer-Verlag, 1984

[56] L.M. Wang, Y. Shen, Q. Yin, G.D. Zhang, Adaptive synchronization of memristor-based neural networks with time-varying delays, IEEE Trans. Neural Netw. Learn. Syst. 26 (2015) 2033-2042. 